\title{
Sampling-based stochastic analysis of the PKN model for hydraulic fracturing
}

\author{
Hasini Garikapati ${ }^{1,2} \cdot$ Clemens V. Verhoosel $^{1} \cdot$ E. Harald van Brummelen ${ }^{1} \cdot$ Sergio Zlotnik ${ }^{2} \cdot$ Pedro Díez $^{2}$
}

Received: 25 July 2018 / Accepted: 2 October 2018 / Published online: 20 November 2018

(C) The Author(s) 2018

\begin{abstract}
Hydraulic fracturing processes are surrounded by uncertainty, as available data is typically scant. In this work, we present a sampling-based stochastic analysis of the hydraulic fracturing process by considering various system parameters to be random. Our analysis is based on the Perkins-Kern-Nordgren (PKN) model for hydraulic fracturing. This baseline model enables computation of high fidelity solutions, which avoids pollution of our stochastic results by inaccuracies in the deterministic solution procedure. In order to obtain the desired degree of accuracy of the computed solution, we supplement the employed time-dependent moving-mesh finite element method with two new enhancements: (i) global conservation of volume is enforced through a Lagrange multiplier; (ii) the weakly singular behavior of the solution at the fracture tip is resolved by supplementing the solution space with a tip enrichment function. This tip enrichment function enables the computation of the tip speed directly from its associated solution coefficient. A novel incremental-iterative solution procedure based on a backward-Euler time-integrator with sub-iterations is employed to solve the PKN model. Direct Monte-Carlo sampling is performed based on random variable and random field input parameters. The presented stochastic results quantify the dependence of the fracture evolution process - in particular the fracture length and fracture opening - on variations in the elastic properties and leak-off coefficient of the formation, and the height of the fracture.
\end{abstract}

Keywords Hydraulic fracturing · Perkins-Kern-Nordgren model · Finite element method · Moving-boundary problem . Stochastic analysis $\cdot$ Random fields $\cdot$ Sensitivity analysis $\cdot$ Monte-Carlo method

Hasini Garikapati

h.garikapati@tue.nl

Clemens V. Verhoosel

c.v.verhoosel@tue.nl

E. Harald van Brummelen

e.h.v.brummelen@tue.nl

Sergio Zlotnik

sergio.zlotnik@upc.edu

Pedro Díez

pedro.diez@upc.edu

1 Eindhoven University of Technology, PO Box 513, 5600 MB, Eindhoven, The Netherlands

2 Universitat Politècnica de Catalunya, Campus Nord, Carrer de Jordi Girona, 1, 3, 08034, Barcelona, Spain

\section{Introduction}

Hydraulic fracturing processes-i.e., the fracturing of rock formations by a pressurized liquid to improve connectivity of reservoirs and geothermal formations-are surrounded by uncertainty, as rock formations are highly heterogeneous and available formation data is limited. Further improvement of models and simulation tools to understand this process is instrumental to increasing operational effectivity and to reliably quantify the risks and uncertainties that are involved.

Hydraulic fracturing models involve the coupling of three sub-models: (i) a solid mechanics model which describes the deformation of the rock formation induced by the fluid load; (ii) a fluid flow model to describe the fracturing fluid in the crack, as well as its leak-off into the rock formation; and (iii) a fracture mechanics model including a fracture propagation criterion. Intrinsic characteristics of such models are the (strong) non-linearities related to the coupling between the solid and fluid, the singularities in the physical fields near the fracture front, the moving (fracture) 
domain boundaries, the degeneration of the governing equations near the tip region, and pronounced multiscale effects with the length scales varying from millimeters for the fracture opening near the tip to kilometers for the length of the fracture.

Various practical model simplifications-most commonly restricting the model to a single two-dimensional planar fracture-have been proposed, the most prominent of which are (i) the Perkins-Kern-Nordgren (PKN) model $[29,31]$ for a fracture of fixed height and elliptical crosssection (leveraging the Sneddon solution for the elasticity problem [37]), which propagates horizontally in one direction; (ii) the radial model for a horizontal Penny-shaped crack that evolves evenly in all directions in accordance with Sneddon's solution [36]; and (iii) the KhristianovicGeertsma-De Klerk (KGD) model [11, 22, 47] for a vertical fracture of fixed height that propagates horizontally in one direction. Various pseudo-three-dimensional (P3D) models [34] and planar-three-dimensional (PL3D) models [2, 35] have been proposed over the years to enable consideration of more complex fracture patterns and fractures in multilayer formations. These P3D models typically extend the abovementioned two-dimensional models by considering a variation of fracture height in combination with fracture length and width. Although these classical models are generally based on restrictive and often ad hoc assumptions, they are still widely used in the industry [19].

Despite the simplifications in the abovementioned models, analytical solutions can only be obtained in limiting cases (e.g., [22, 29, 47]). General solution strategies for these models rely on the use of computational techniques. Versatile and reliable simulators for hydraulic fracturing processes are indispensable in gaining further understanding of the process, in particular because direct observation possibilities (typically several kilometers below the earth surface) are limited. The most prominent computational techniques used for hydraulic fracturing are finite volume methods (e.g., [30]), finite element methods (e.g., [4, 28, 40]), boundary integral methods (e.g., [9]) and discrete element methods (e.g., [26]). Recent advancements in numerical methods for hydraulic fracturing that are particularly noteworthy are the eXtended Finite Element Techniques (X-FEM) (e.g., [12, 20, 32, 33]) and phase-field methods (e.g., [24, 25, 42, 43]).

Although the importance of considering realistic geological situations is acknowledged [7], hydraulic fracture evolution in formations with uncertain heterogeneous rock properties (e.g., elastic moduli, compression/tensile strength, porosity, permeability) have not been studied in detail. In [46], a reliability analysis is conducted using analytical models for hydraulic fracturing. The possibility of considering stochastic heterogeneities in combination with computational models has been explored in [44], where the initiation and evolution of fracture has been studied using MonteCarlo sampling. The main challenge in such studies relates to the computational feasibility, in the sense that the computational effort of the deterministic simulations (whose error must be controlled in relation to the stochastic variations) is prohibitive in the context of sampling-based stochastic techniques.

In this work, we present a detailed probabilistic analysis of the hydraulic fracturing process based on MonteCarlo simulations. The computational tractability of the stochastic framework considered herein motivates the use of the two-dimensional PKN model. We represent the (epistemic) uncertain parameters of the PKN model as random variables and/or random fields, and investigate the influence of these uncertain input parameters on the fracture geometry (in particular the fracture length and opening at the well bore). As part of the stochastic analysis, we present a sensitivity analysis of the deterministic model. It is worth mentioning that similar analyses can be found in the literature for the deterministic setting [14, 45]. The primary focus of this work is the direct analysis of the propagation of heterogeneous uncertainties in the hydraulic fracturing process. As part of this study, we present a detailed derivation of the PKN model in the context of random (spatially correlated) heterogeneous data. Control over accuracy of the numerical approximation of the PKN model is of paramount importance, because excessive numerical errors would pollute the stochastic uncertainty quantification. In this regard, we propose to use two new features in the numerical method for the PKN model to control its accuracy: (i) a Lagrange multiplier method to enforce the conservation of volume and (ii) a special enrichment function for the finite element discretization of the PKN model to overcome tip singularity issues.

In Section 2, the governing equations are discussed, with a special focus on the incorporation of rock heterogeneities in the PKN model. In Section 3, the weak formulation and finite element discretization of the model are presented including various algorithmic details. The stochastic setting and Monte-Carlo method are introduced in Section 4, where the random field discretization of the heterogeneous properties using the Karhunen-Loève expansion is also discussed. Numerical simulations are presented in Section 5 to study the influence of input uncertainties in hydraulic fracturing. Finally, conclusions are presented in Section 6.

\section{The PKN model for hydraulic fracturing}

In this section, we review the PKN model for hydraulic fracturing in the context of stochastic analyses with heterogeneous random fields. The PKN model-which was originally formulated by Perkins and Kern [31] 
and later amended with a leak-off model by Nordgren [29] and a propagation condition by Kemp [16]-is a practical candidate for preliminary studies of the probabilistic behavior of hydraulic fracturing by virtue of its computational tractability. Although highly simplified, the PKN model is based on fundamental physical principles and is capable of generating practically meaningful results [39].

\subsection{Problem definition}

The key assumption of the PKN model is that it considers a planar fracture with a constant height $H$ (see Fig. 1). Displacements and displacement gradients in the surrounding solid are assumed to remain small, and the material is assumed to be linear elastic and isotropic. The fracture surface resides in the $x y$-plane, while the fracture opens in the $z$-direction. The fracture aperture in the fracture plane is denoted by $w(x, y, t)$, and the aperture at the $y=0$ center line by $\hat{w}(x, t)$. The fracture connects to the well at $x=0$ and its evolving front is situated at $x=L(t)$.

A Newtonian fluid is injected into the fracture at the well with a controlled flow rate $i(t)$, and the flow inside the permeable crack is assumed to be laminar. The fracture process is assumed to be in the viscosity-based regime, where toughness effects can be neglected (propagation is governed by friction and leak-off effects). At the front of the fracture, a fluid lag is assumed to be zero, i.e., the fracture front coincides with the fluid front. Moreover, a spurt losses due to the creation of new fracture surfaces (see e.g., [39]) that are ignored.

\subsection{Governing equations}

In this section, the governing equations of the sub-models are reviewed. In the presented derivations, we focus on those aspects of the sub-models that need careful consideration in the context of the stochastic analysis discussed in the remainder of this work.

\subsubsection{Fluid flow model}

The PKN model is based on the conservation of mass of the fluid, which establishes a link between the injected volume, the created fracture volume and the leak-off volume. The differential material balance for the fracturing fluid is given by the following:

$\frac{\partial q}{\partial x}+\frac{\partial A}{\partial t}=-s_{c_{l}}$

for all $x \in(0, L(t))$, where $q(x, t)$ is the volume rate of flow through the cross-sectional area $A(x, t)=$ $\int_{-H / 2}^{H / 2} w(x, y, t) \mathrm{d} y$ and $s_{c_{l}}(x, t)$ is the rate of fluid volume loss per unit length of the fracture. At the well $(x=0)$ the flow rate is equal to the injection rate according to $q(0, t)=i(t)$.

The flow rate inside the fracture is related to the pressure gradient by assuming Poiseuille flow [48]. For such a flow, the advective terms are assumed to remain small, so that the incompressible Navier-Stokes equations reduce to the Stokes equations. The PKN model moreover assumes a horizontal slot flow [6] with a parabolic fluid velocity profile as follows:

$v=-\frac{1}{2 \mu_{f}} \frac{\partial p}{\partial x}\left(\frac{w^{2}}{4}-z^{2}\right)$

where $w(x, y, t)$ is the opening of the fracture and $\mu_{f}$ is the fluid viscosity. As will be discussed in more detail in the context of the solid model (Section 2.2.2), the assumptions of the PKN model lead to an ellipsoidal cross-section. The fracture aperture is then given by the following:

$w=\hat{w} \sqrt{1-4 y^{2} / H^{2}}$,

where $\hat{w}(x, t)$ is the maximum aperture at $y=0$. The cross-sectional area is $A(x, t)=\frac{\pi}{4} H \hat{w}(x, t)$ and the fluid flow follows by integration of the fluid velocity in Eq. 2 as follows:

$$
\begin{aligned}
q & =\int_{-H / 2}^{H / 2} \int_{-w / 2}^{w / 2} v \mathrm{~d} y \mathrm{~d} z=-\frac{1}{12 \mu_{f}} \frac{\partial p}{\partial x} \int_{-H / 2}^{H / 2} w^{3} \mathrm{~d} y \\
& =-\frac{\pi H \hat{w}^{3}}{64 \mu_{f}} \frac{\partial p}{\partial x} .
\end{aligned}
$$

The leak-off volume rate per unit length of fracture in Eq. 1 follows the phenomenological law proposed by Carter [15]:

$s_{c_{l}}=\frac{2 H c_{l}}{\sqrt{t-\tau}}$

In this expression, $c_{l}$ is the leak-off coefficient and $\tau(x)$ is the arrival time of the fracture tip at location $L$, i.e., $\tau(x)=$ $L^{-1}(x)$. The main assumptions behind this model are that (i) the fracturing fluid deposits a thin layer of relatively low permeability material (known as the filter cake) on the inner faces of the fracture, with the deposition rate being proportional to the leak-off rate, and (ii) the viscosity of the filtrate is high enough to fully displace the fluid already present in the rock pores.

Substitution of Eqs. 4 and 5 in the material balance (1) then yields the fluid flow mass balance for all $x \in(0, L(t))$ :

$$
\frac{\pi H}{64 \mu_{f}} \frac{\partial}{\partial x}\left(\hat{w}^{3} \frac{\partial p}{\partial x}\right)=\frac{2 H c_{l}}{\sqrt{t-\tau(x)}}+\frac{\pi H}{4} \frac{\partial \hat{w}}{\partial t}
$$

\subsubsection{Solid deformation model}

To derive the relation between the fluid pressure and the solid deformation as used in the PKN model, we consider the infinite domain $\Omega=R_{+} \times R \times R$ with material 


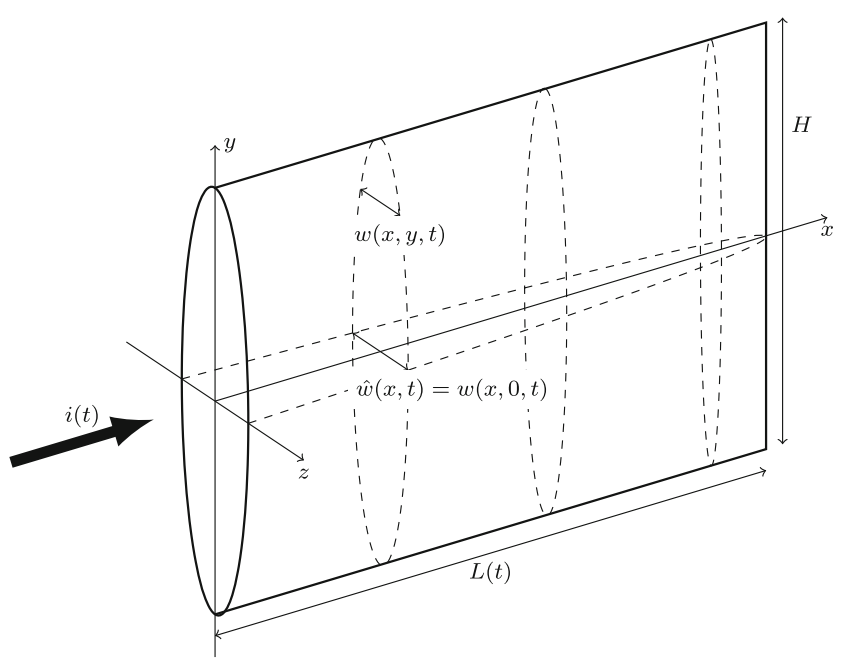

Fig. 1 Schematic representation of the fracture geometry and boundary conditions for the PKN model. Note that both the fracture aperture, $w$, and the fracture length, $L$, are time-dependent

coordinates $\boldsymbol{x}=(x, y, z)$ and an evolving fracture surface (see Fig. 1):

$\Gamma_{c}(t)=\left\{x \in \Omega \mid x \leq L(t),-\frac{H}{2} \leq y \leq \frac{H}{2}, z=0\right\}$

Assuming inertia and gravity effects to be negligible, the solid deformation, $u=(\xi, \eta, \zeta)$, follows from the momentum balance as follows:

$\nabla \cdot \sigma=0 \quad$ in $\Omega$,

where $\sigma$ is the Cauchy stress tensor. The Cauchy stress follows Hooke's law for isotropic materials as follows:

$\sigma=2 \mu_{s} \varepsilon+\lambda_{s} \operatorname{tr}(\varepsilon) I$

where $\varepsilon=\nabla^{\mathrm{s}} u$ is the infinitesimal strain tensor. In the context of the stochastic analysis considered herein, the Lamé parameters $\mu_{s}(x)$ and $\lambda_{s}(x)$ are heterogeneous fields directly related to the Young's modulus, $E(x)$, and Poisson's ratio, $v(x)$. Under the horizontal slot flow assumption, the viscous contribution to the normal stress along the fracture surface vanishes, and the solid is loaded by the fluid pressure, i.e., $\sigma n=-p n$ on $\Gamma_{c}$ where $n$ is the normal vector internal to the fluid domain.

An important aspect of the PKN model is that it relies on a planar deformation solid mechanics submodel, which provides a local relation between the pressure and the fracture aperture. This reduction of the elasticity problem (8) - which in the literature is generally considered with homogeneous material properties-is key to the computational tractability of the PKN model, but evidently hinges on the postulation of additional modeling assumptions, viz.: (i) The deformations are planar, in the sense that the solid does not deform in the direction of the fracture propagation $(\xi=0)$, and that a plane strain condition in that direction applies $\left(\varepsilon_{\mathrm{xx}}=\varepsilon_{\mathrm{xy}}=\varepsilon_{\mathrm{xz}}=0\right)$; (ii) heterogeneous variations perpendicular to the fracture propagation direction are assumed to be negligible, i.e., the model parameters are independent of the $y$ - and $z$ coordinates.

The assumptions that lead to the local solid mechanics model evidently restrict the applicability of the model. Even in the generally considered case of heterogeneous material properties, the planarity assumption is debatable in the vicinity of the fracture tip, where both the geometry and pressure loading vary significantly in the $x$-direction (see, e.g., [3]). Although highly simplified, the local elasticity model is widely recognized to yield meaningful results in a variety of simulation scenarios [39]. An important point to make in the context of this contribution is that we consider the material properties to be random fields (see Section 4), which are parametrized by a mean value, a standard deviation, and an auto-correlation length, $\ell$. This auto-correlation length is a measure of the correlation between any two material points in a random field, where a large correlation length implies that the spatial frequency of the heterogeneous field is low. In order for the planarity assumption to be meaningful, we consider sufficiently large auto-correlation lengths. More specifically, high wavenumber variations in the material properties along the $x$-axis are not considered herein. Moreover, the heterogeneities in the planes perpendicular to the $x$-direction are assumed to be negligible, at least in the sense that their influence is averaged out when considering the integrated opening of the fracture. In line with these assumptions, in this manuscript, we only consider random heterogeneities in the direction of propagation with sufficiently large auto-correlation lengths.

Under the assumptions discussed above, the deformation in an arbitrary plane perpendicular to the $x$-direction can be deduced from the momentum balance (8) and constitutive relation (9), which, written out in components, yields:

$$
\begin{aligned}
& 2 \mu_{s}(x) \frac{\partial^{2} \eta}{\partial y^{2}}+\lambda_{s}(x)\left(\frac{\partial^{2} \eta}{\partial y^{2}}+\frac{\partial^{2} \zeta}{\partial y \partial z}\right)+\mu_{s}(x)\left(\frac{\partial^{2} \eta}{\partial z^{2}}+\frac{\partial^{2} \zeta}{\partial y \partial z}\right)=0 \\
& \mu_{s}(x)\left(\frac{\partial^{2} \eta}{\partial y \partial z}+\frac{\partial^{2} \zeta}{\partial y^{2}}\right)+2 \mu_{s}(x) \frac{\partial^{2} \zeta}{\partial z^{2}}+\lambda_{s}(x)\left(\frac{\partial^{2} \eta}{\partial y \partial z}+\frac{\partial^{2} \zeta}{\partial z^{2}}\right)=0
\end{aligned}
$$

Supplemented with the boundary conditions $\sigma_{\mathrm{zz}}(x)=$ $-p(x) n_{z}$ and $\sigma_{\mathrm{yz}}=0$ on the fracture boundary and vanishing far field conditions, this problem can be solved analytically. The fracture opening in the case of a constant pressure in the $y z$-plane is given by (see e.g., Lowengrub [21] and Sneddon [37] for details)

$$
\begin{aligned}
w(x, y) & =4 p(x) \int_{y}^{H / 2} \frac{\bar{y}\left(1-v(x)^{2}\right)}{E(x) \sqrt{\bar{y}^{2}-y^{2}}} \mathrm{~d} \bar{y} \\
& =\frac{2 H p(x)}{E^{\prime}(x)} \sqrt{1-4 y^{2} / H^{2}} \quad|y| \leq \frac{H}{2},
\end{aligned}
$$


where $E^{\prime}(x)=E(x) /\left(1-v(x)^{2}\right)$ is the plane strain modulus, which is heterogeneous only in the $x$-direction. We note that the elliptical profile in Eq. 3 is a direct result of the setting of the elasticity problem considered here, with the maximum aperture equal to

$\hat{w}(x)=\frac{2 H p(x)}{E^{\prime}(x)}$.

An essential property of this solution is that along the crack path the fracture aperture is linearly related to the pressure. The local nature of this relation is a direct consequence of the assumed planar deformation. Note that the stress field and displacement field can be derived in the form of integral representations [8].

\subsubsection{Fracture propagation model}

In the PKN model, it is assumed that once the fracture has exceeded a certain distance, the energy dissipation associated with the fracture of the rock material is small compared to energy dissipation associated with the viscous flow of the fracturing fluid. This effectively neglects the fracture toughness, and fracture propagation is purely driven by the fluid velocity. Herein, we adopt the standard assumption of zero fluid lag [29]-i.e., the velocity of the fluid at the fluid front and the tip propagation speed are equal-so that tip propagation follows the Stefan condition

$v(L(t), t)=\lim _{x \rightarrow L(t)} \frac{q(x, t)}{A(x, t)}=\dot{L}(t)$.

Substitution of the flow rate (4) and surface area then yields:

$\dot{L}(t)=-\frac{1}{16 \mu_{f}} \lim _{x \rightarrow L(t)} \hat{w}^{2} \frac{\partial p}{\partial x}$

The limits in Eqs. 13 and 4 are one-sided, from below.

\subsection{The coupled initial boundary value problem}

The hydraulic fracture problem is characterized by a strong coupling of the sub-models discussed above. The solid deformation is coupled to the fluid through the pressure loading along the fracture surface, while the fluid depends on the fracture opening though the Poiseuille flow profile. The fracture propagation condition is coupled directly to the fluid flow through the Stefan condition (13), and in turn influences the fluid flow by virtue of the fact that fracture propagation extends the fluid flow domain. The pointwise relation between pressure and the fracture opening in Eq. 12 allows the formulation of a singlefield free-boundary problem. Herein, we consider the initial boundary value problem for the fracture opening on the time interval $(0, T) \ni t$ with evolving domain $(0, L(t)) \ni x$ :

$$
\begin{cases}\frac{\pi}{128 \mu_{f}} \frac{\partial}{\partial x}\left(\hat{w}^{3} \frac{\partial\left(E^{\prime} \hat{w}\right)}{\partial x}\right)=\frac{2 H c_{l}}{\sqrt{t-\tau}}+\frac{\pi H}{4} \frac{\partial \hat{w}}{\partial t} & \forall x \in(0, L(t)), \forall t \in \times(0, T) \\ -\left.\frac{\pi}{128 \mu_{f}}\left(\hat{w}^{3} \frac{\partial\left(E^{\prime} \hat{w}\right)}{\partial x}\right)\right|_{x=0}=i(t) & \forall t \in(0, T) \\ \hat{w}(L(t), t)=0 & \forall t \in(0, T) \\ \hat{w}(x, 0)=0 & \forall x \in\left(0, L_{0}\right) \\ \dot{L}(t)=-\left.\frac{1}{96 \mu_{f} H} \frac{\partial\left(E^{\prime} \hat{w}^{3}\right)}{\partial x}\right|_{x=L(t)} & \forall t \in(0, T) \\ L(0)=L_{0} & \end{cases}
$$

Note that the omission of fluid lag in the model results in the tip boundary condition $\hat{w}(L(t), t)=0$, reflecting zero fracture opening at the tip. This boundary condition leads to singular behavior of the fracture opening (and pressure) at the tip, which is an important characteristic of the coupled problem (15a-f). In Refs. [1, 10, 19], it is shown that in the viscosity-dominated regime, the toughness of the solid is small enough that the solution of a hydraulic fracture can be approximated by the zero toughness solution and the aperture solution in the proximity of the tip is proportional to:

$$
\hat{w}(x, t) \propto \sqrt[3]{L(t)-x}
$$

We note that, due to the nature of this singularity, and assuming a finite plane strain modulus, the tip propagation relation $((15 \mathrm{e}))$ results in a finite propagation speed.

\section{Deterministic computational methodology}

In this section, we present a methodology that enables the computation of solutions of the PKN model with an accuracy that makes it suitable for conducting a sampling-based stochastic analysis. In Section 3.1, we first discuss the incremental-iterative solution procedure, which is used to integrate the time-dependent moving-boundary 


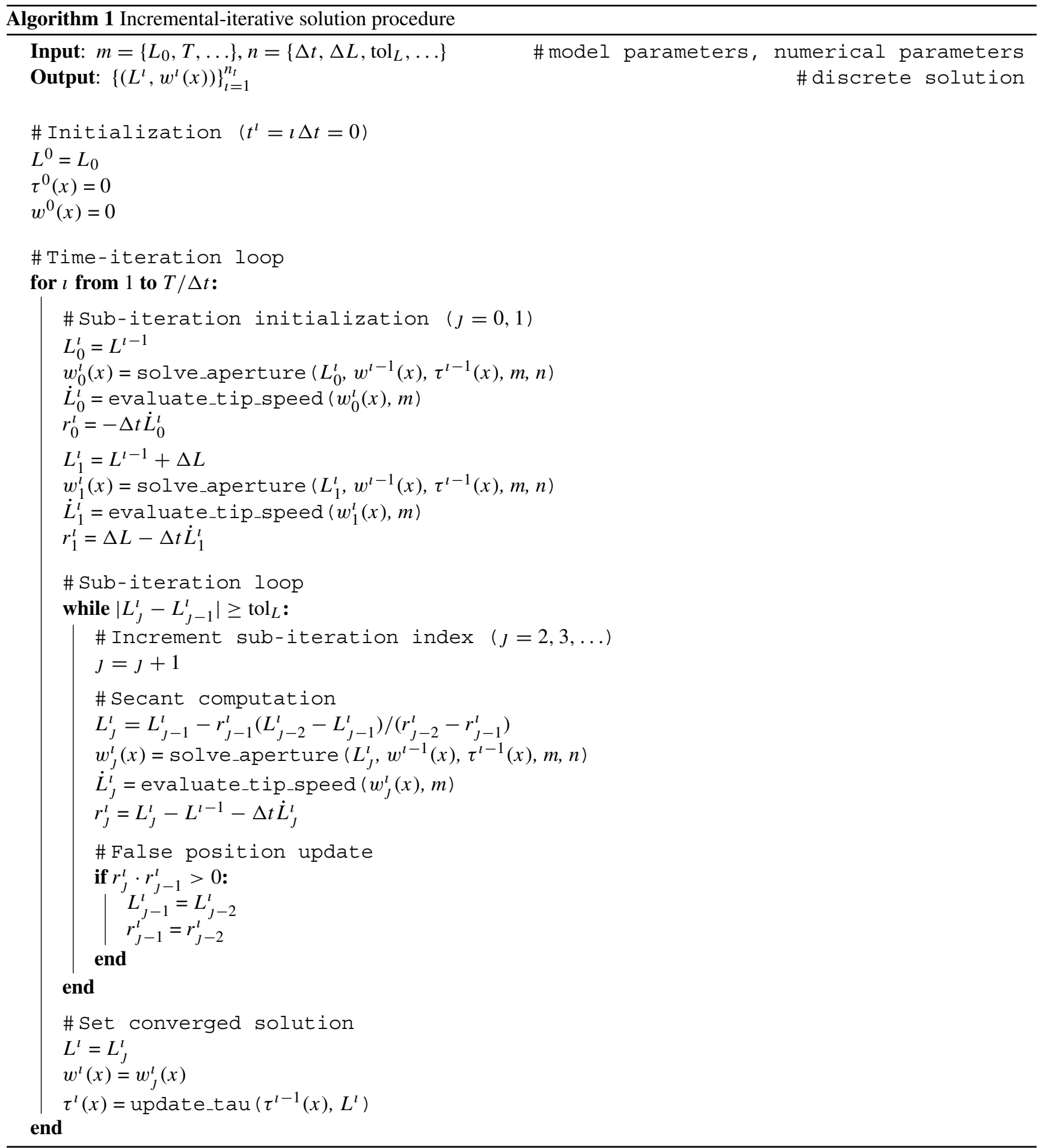

problem. In Section 3.2, we then discuss the spatial finite element discretization of the nonlinear system of equations introduced above, including two essential enhancements, viz. incorporation of a Lagrange multiplier to enforce the volume-conservation constraint, and a solution space enrichment to resolve the singularity at the fracture tip.

\subsection{Incremental-iterative solution procedure}

To solve the time-dependent moving-boundary problem (15a-f), we employ the incremental-iterative solution procedure outlined in Algorithm 1. We denote the time step size and index by $\Delta t$ and $\imath=0, \ldots, n_{t}$, respectively, such that $t^{l}=\imath \Delta t$ and $T=n_{t} \Delta t$. The solution at time step $\iota$ and 
sub-iteration $J=0,1,2, \ldots$ is written as $w_{j}^{l}(x)$ and $L_{j}^{l}$. The sub-iteration index $J$ is omitted for converged solutions, i.e., $w^{l}(x)$ and $L^{l}$.
We consider the implicit time integration of both the fracture aperture and the fracture length, such that the coupled system $(15 \mathrm{a}-\mathrm{f})$ is discretized in time as follows:

$$
\left\{\begin{array}{l}
\frac{\pi}{128 \mu_{f}} \frac{\partial}{\partial x}\left(\left(\hat{w}^{l}\right)^{3} \frac{\partial\left(E^{\prime} \hat{w}^{l}\right)}{\partial x}\right)=\frac{2 H c_{l}}{\sqrt{t^{l}-\tau^{l-1}}}+\frac{\pi H}{4} \frac{\hat{w}^{l}-\hat{w}^{l-1}}{\Delta t} \quad \forall x \in(0, L(t)) \\
-\left.\frac{\pi}{128 \mu_{f}}\left(\left(\hat{w}^{l}\right)^{3} \frac{\partial\left(E^{\prime} \hat{w}^{l}\right)}{\partial x}\right)\right|_{x=0}=i\left(t^{l}\right) \\
\hat{w}^{l}\left(L^{l}\right)=0 \\
\frac{L^{l}-L^{l-1}}{\Delta t}=-\left.\frac{1}{96 \mu_{f} H} \frac{\partial\left(E^{\prime}\left(\hat{w}^{l}\right)^{3}\right)}{\partial x}\right|_{x=L^{l}}
\end{array}\right.
$$

for all $\iota=1, \ldots, n_{t}$ with initial conditions $\hat{w}^{0}(x)=0$ and $L^{0}=L_{0}$.

To solve this moving-boundary problem at time step $\imath=$ $1, \ldots, n_{t}$, within each time step, we sub-iterate between the aperture problem (17a)-(17c) and the propagation problem (17d) until convergence is achieved. The solution of the aperture problem (solve_aperture in Algorithm 1) is approximated using a finite element discretization in combination with a Newton-Raphson procedure to resolve the non-linearity. Details regarding the finite element discretization will be discussed in Section 3.2. The propagation problem is solved by using the regula falsi method to find the root of the residual function

$r_{j}^{l}=r\left(L_{j}^{l}\right)=L_{j}^{l}-\left(L^{l-1}+\Delta t \dot{L}_{j}^{l}\right)$.

Given an iterate for the fracture length, $L_{j}^{l}$, the corresponding fracture aperture $w_{j}^{l}$ is computed using the solve_aperture procedure, after which the procedure evaluate_tip_speed is called to determine the associated tip speed in accordance with the following:

$\dot{L}_{J}^{\imath}=-\left.\frac{1}{96 \mu_{f} H} \frac{\partial\left(E^{\prime}\left(\hat{w}_{J}^{l}\right)^{3}\right)}{\partial x}\right|_{x=L_{J}^{l}}$

The regula falsi procedure is initialized with the fracture length at the previous time step, $L_{0}^{l}=L^{l-1}$, and with the forced propagation, $L_{1}^{l}=L^{l-1}+\Delta L$. We note that the residual $r_{0}^{l}=-\Delta t \dot{L}_{j}^{l}$ is non-negative as a consequence of the non-negativity of the propagation speed. The residual $r_{1}^{l}=\Delta L-\Delta t \dot{L}_{j}^{l}$ is forced to be positive by selecting the numerical parameter $\Delta L>0$ to be sufficiently large. For all simulations in Section 5 , we set $\Delta L$ equal to the element size at the fracture tip, and take a time step that is sufficiently small to ensure positivity of the residual $r_{1}^{l}$.

The sub-iteration procedure is terminated when the fracture length converges to a specified tolerance, i.e., $\left|L_{j}^{l}-L_{j-1}^{l}\right|<\operatorname{tol}_{L}$. The converged iterates $\hat{w}_{j}^{l}(x)$ and $L_{j}^{l}$ for the fracture aperture and length are then used as the initial conditions for the next time step. The fracture arrival function $\tau^{l}(x)$ for the next time step is updated in the update_tau routine. Since the arrival time function is evaluated by linear interpolation in the list $\left\{\left(L^{\hat{\imath}}, t^{\hat{\imath}}\right)\right\}_{\hat{\imath}=0}^{l}$, this update routine merely appends the converged fracture length to this list. Note that we assume zero leak-off in the initial crack, so that we do not need to evaluate the arrival function for $x \leq L^{0}$. For $x>L^{l-1}$ the arrival time is taken as the time at which the crack reached $L^{l-1}$. As a result $t^{l}-\tau^{l-1}(x) \geq \Delta t$, everywhere in the interval $\left(L^{0}, L^{l}\right)$ which effectively bypasses the occurrence of a singularity in the leak-off term at the fracture tip.

\subsection{Finite element discretization}

The solve_aperture routine uses the finite element method to compute the fracture aperture $\hat{w}_{j}^{l}$ based on the fracture length $L_{j}^{l}$ and the aperture and arrival time at the previous time step, $w^{l-1}$ and $\tau^{l-1}$, respectively. To derive the finite element formulation, the weak form of Eq. $17 \mathrm{a}-\mathrm{d}$ is considered, where the sub-iteration index has been dropped for notational convenience:

$$
\begin{aligned}
& \text { Find } \hat{w}^{l} \in \mathcal{V}^{l} \text { such that: } \\
& \begin{array}{l}
\frac{\pi}{4} \int_{0}^{L^{l}}\left(\frac{\left(\hat{w}^{l}\right)^{3}}{32 \mu_{f}} \frac{\partial\left(E^{\prime} \hat{w}^{l}\right)}{\partial x} \frac{\partial v}{\partial x}+\frac{H}{\Delta t} \hat{w}^{l} v\right) \mathrm{d} x= \\
\frac{\pi H}{4 \Delta t} \int_{0}^{L^{l-1}} \hat{w}^{l-1} v \mathrm{~d} x-2 H c_{l} \int_{L_{0}}^{L^{l}} \frac{v}{\sqrt{t^{l}-\tau^{l-1}}} \mathrm{~d} x+i\left(t^{l}\right) v(0) \quad \forall v \in \mathcal{V}^{l}
\end{array}
\end{aligned}
$$


The time-dependent test and trial space $\mathcal{V}^{l}$ specified below are defined such that the Dirichlet boundary conditions at the tip are satisfied, and all integrals in the above formulation are bounded. Note that the right-hand-side integral involving the fracture aperture at the previous time step is computed over the fracture domain at the previous time step, which reflects the fact that ahead of the fracture tip the aperture is equal to zero. Moreover, the initial crack is excluded from the integration domain for the right-handside term involving the leak-off, which results from the assumption that there is no leak-off in the initial crack.

The weak formulation (20) is discretized using the finite element method by approximating the maximum aperture as follows:

$\hat{w}^{l, h}(x)=\sum_{i \in \mathcal{I}^{l}} N_{i}^{l}(x) a_{i}^{l}$,

where the index set $\mathcal{I}^{l}$ contains the indices of the shape functions $\left\{N_{i}\right\}_{i \in \mathcal{I}^{l}}$ constructed over a mesh $\mathcal{T}^{l}$ that partitions the evolving domain $\left(0, L^{l}\right)$. The corresponding discrete trial and test space are given by $\mathcal{V}^{l, h}=$ $\operatorname{span}\left(\left\{N_{i}\right\}_{i \in \mathcal{I}^{l}}\right) \subset \mathcal{V}^{l}$.

The finite element discretization considered in this work is based on linear Lagrange finite elements, where the linear basis function associated with the tip node is constrained in accordance with the zero-aperture Dirichlet condition at the tip. Because of the nature of the solution, we grade the mesh toward the tip by specifying the element size at the tip, the increase ratio between two adjacent elements, and the maximal element size that is approached toward the inflow boundary. A schematic representation of such a graded mesh is shown in Fig. 2. Note that the first right-hand-side integral term in Eq. 20 involves the product of functions defined on two different meshes, and hence requires evaluation on an overlay mesh.

A discretization of the problem (20) based on linear finite elements-even though graded toward the tip-leads to an unacceptable loss of accuracy at meshes that are computationally tractable within the scope of this work. This performance deterioration is a consequence of (i) the flux (4) being highly non-linearly dependent on the fracture aperture and (ii) the singular behavior at the tip not being represented by the linear finite element basis. In the remainder of this subsection, we propose two enhancements to ameliorate this performance degradation. In Section 5.1, the numerical performance of these enhancements will be assessed in the scope of a benchmark simulation.

\subsubsection{Mass conservation constraint}

Although the weak formulation (20) is consistently derived from the mass balance (6), the finite element approximation of Eq. 20 does not strictly comply with the local mass balance and its integrated global version. Since adequate representation of the conservation of mass is of critical importance for obtaining accurate solutions, we herein propose to explicitly enforce global conservation in our approximation. We obtain the global balance of mass by integration of the time-discrete version of Eq. 6 over the entire domain:

$$
\begin{aligned}
i\left(t^{l}\right)= & 2 H c_{l} \int_{L_{0}}^{L^{l}} \frac{1}{\sqrt{t^{l}-\tau^{l-1}}} \mathrm{~d} x+\frac{\pi H}{4 \Delta t} \int_{0}^{L^{l-1}} \hat{w}^{l}-\hat{w}^{l-1} \mathrm{~d} x \\
& +\frac{\pi H}{4 \Delta t} \int_{L^{l-1}}^{L^{l}} \hat{w}^{l} \mathrm{~d} x
\end{aligned}
$$

This global balance clearly shows that the injected volume is conserved by leak-off through the fracture (first term), fracture opening (second term), and fracture propagation (third term). We explicitly enforce the global conservation law (22) in the weak formulation (20) by means of a Lagrange multiplier, $\Lambda^{l}$ :

$$
\left\{\begin{array}{cl}
\text { Find } \hat{w}^{l} \in \mathcal{V}^{l} \text { and } \Lambda^{l} \in R \text { such that: } & \\
\frac{\pi}{4} \int_{0}^{L^{l}}\left(\frac{\left(\hat{w}^{l}\right)^{3}}{32 \mu_{f}} \frac{\partial\left(E^{\prime} \hat{w}^{l}\right)}{\partial x} \frac{\partial v}{\partial x}+\frac{H}{\Delta t} \hat{w}^{l} v\right) \mathrm{d} x-\Lambda^{l}\left(\frac{\pi H}{4 \Delta t} \int_{0}^{L^{l}} v \mathrm{~d} x\right)= & \forall v \in \mathcal{V}^{l} \\
\frac{\pi H}{4 \Delta t} \int_{0}^{L^{l-1}} \hat{w}^{l-1} v \mathrm{~d} x-2 H c_{l} \int_{L_{0}}^{L^{l}} \frac{v}{\sqrt{t^{l}-\tau^{l-1}}} \mathrm{~d} x+i\left(t^{l}\right) v(0) & \\
\Psi\left(i\left(t^{l}\right)-2 H c_{l} \int_{L_{0}}^{L^{l}} \frac{1}{\sqrt{t^{l}-\tau^{l-1}}} \mathrm{~d} x-\frac{\pi H}{4 \Delta t} \int_{0}^{L^{l-1}} \hat{w}^{l}-\hat{w}^{l-1} \mathrm{~d} x-\frac{\pi H}{4 \Delta t} \int_{L^{l-1}}^{L^{l}} \hat{w}^{l} \mathrm{~d} x\right)=0 & \forall \Psi \in R
\end{array}\right.
$$

\subsubsection{Singular tip enrichment}

As discussed in Section 2.3, the aperture solution to the problem $(15 \mathrm{a}-\mathrm{f})$ is singular at the tip in accordance with Eq. 16. Evidently, this singular solution behavior is approximated poorly by the linear finite element basis. As a matter of fact, when solely using the linear finite element basis, the tip propagation speed will always vanish when 
Fig. 2 Schematic representation of the time-dependent graded finite element mesh with a linear finite element basis and a tip enrichment function

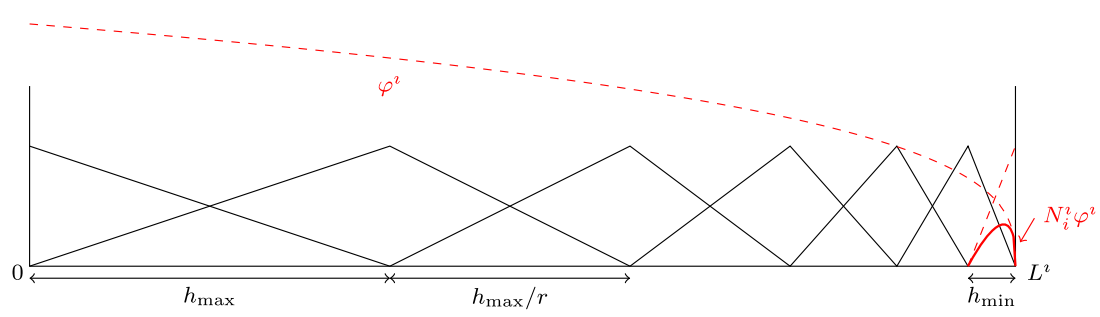

evaluated through Eq. 15e. To improve the finite element approximation, we enrich the test and trial space $\mathcal{V}^{l}$ with the tip asymptote (16):

$\varphi^{l}(x)=\sqrt[3]{L^{l}-x}$

We localize this enrichment function to the tip region using the partition-of-unity method (see, e.g., [23]). The enriched finite element interpolation of the aperture is then given by the following:

$\hat{w}^{l, h}(x)=\sum_{i \in \mathcal{I}^{\imath}} N_{i}^{l}(x) a_{i}^{l}+\sum_{j \in \mathcal{J}^{l}} N_{j}^{l}(x) \varphi^{l}(x) b_{j}^{l}$

where the index set $\mathcal{J}^{l} \subset \mathcal{I}^{l}$ contains the indices of the nodes that are enriched. In the numerical simulations considered in Section 5, we only enrich the linear finite element function associated with the tip, which we have found to be effective. A schematic representation of this enrichment is shown in Fig. 2.

\section{Stochastic setting}

In this section, we introduce the stochastic setting of the PKN problem. Section 4.1 introduces the Monte-Carlo method that we use in this work. In Section 4.2, we discuss the random variables and random fields for the model parameters. We do this in an abstract setting, where we denote the set of model parameters by $m=\left\{m^{1}, m^{2}, \ldots\right\}$. For a given set of model parameters, we can compute the response of the system, which is characterized by the fracture length $L(t)$ and space-time-dependent fracture aperture function $\hat{w}(x, t):[0, L(t)] \rightarrow R$ defined over the moving domain. From this response, we can deduce the observable parameters as $d=\left\{d^{1}, d^{2}, \ldots\right\}$. In the remainder of this work, we will consider some of the model parameters to be uncertain, viz. the plane strain modulus $\tilde{E}^{\prime}$, the leak-off coefficient, $\tilde{c_{l}}$, and the fracture height $\tilde{H}$. We use the tilde diacritic to indicate that these parameters are stochastic. As observable parameters, we will focus on the final fracture length, $\tilde{L}(T)$, and the maximum fracture mouth opening, $\tilde{\hat{w}}(T)$.

\subsection{Direct Monte-Carlo sampling}

In this work, we use direct Monte-Carlo sampling to compute the stochastic response of the PKN model. The primary reason for using direct Monte-Carlo sampling is that it does not pose any restrictions on the distributions of the model parameters and the observables. Moreover, the non-intrusive character of the method enables its direct application to the PKN model. More advanced stochastic techniques, such as perturbation methods or spectral methods (see [13] for an overview) can aid in reducing the computational effort of the stochastic problem, but application of such techniques to the highly non-linear moving-boundary problem considered here is non-standard and beyond the scope of the current work.

We denote a realization, or sample, of the random set of model parameters $\tilde{m}$ by $m_{k}$, where the subscript $k$ is the index of the sample. The direct Monte-Carlo method generates a sequence of model parameter realizations, $\left\{m_{1}, m_{2}, \ldots, m_{N}\right\}$, and applies the model to construct the corresponding sequence of observables, $\left\{d_{1}, d_{2}, \ldots, d_{N}\right\}$, where $N$ denotes the number of samples. An estimate of the stochastic set of observables, $\tilde{d}$, can then be obtained through statistical analysis of the sequence of samples. In particular, the mean and standard deviation of an observable, $\tilde{d}^{i}$, are computed by the estimators as follows:

$\mu_{d^{i}} \approx \frac{1}{N} \sum_{k=1}^{N} d_{k}^{i}, \sigma_{d^{i}} \approx \sqrt{\frac{1}{N-1} \sum_{k=1}^{N}\left(d_{k}^{i}-\mu_{d^{i}}\right)^{2}}$,

where the symbols $\mu_{d^{i}}$ and $\sigma_{d^{i}}$ denote the mean and standard deviation, respectively. Evidently, the accuracy of the estimators depends on the number of samples $N$. Given a confidence level $C_{\mu}$ for the estimated mean $\mu$ (omitting the subscript $d^{i}$ for notational convenience) - meaning that the estimated mean has a relative accuracy of $\pm\left(1-C_{\mu}\right) / 2$ with probability $C_{\mu}$-the minimal number of samples can be estimated by $[17,27]$ the following:

$N \gtrsim\left(\frac{\Phi^{-1}\left(\frac{1+C_{\mu}}{2}\right)}{1-C_{\mu}}\right)^{2} V_{d^{i}}^{2}$,

where $\Phi$ is the cumulative density function of a standard normal random variable, and $V_{d^{i}}=\sigma_{d^{i}} / \mu_{d^{i}}$ is the 
coefficient of variation of the random observable $\tilde{d}^{i}$. A rough estimate of this coefficient of variation can be obtained using a Monte-Carlo simulation with a small number of samples.

From Eq. 27, it becomes apparent that a draw-back of the direct Monte-Carlo method is the slow convergence of the sampling error (an increase in confidence level) with increase in the number of samples. In the context of computational models, such as that considered in this work, this practically means that the stochastic simulations are computationally very intensive in the case of practically meaningful confidence levels. The fact that Monte-Carlo sampling is non-intrusive-in the sense that it is a method that does not interfere with the deterministic model-makes parallelization possible. We have implemented a parallel master/slave algorithm for our simulations, which shows excellent scalability.

\subsection{Random variable and random field parametrization}

In this work, we represent the considered scalar model parameters, $\tilde{m}^{i}$, by means of log-normal distributions, which are parametrized by the mean value and the standard deviation. Log-normal distributions are considered to avoid physically impossible negative realizations. We employ standard random number generators [5] to obtain the sequence of samples required for Monte-Carlo sampling.

In the case of heterogeneous random fields, $\tilde{m}^{i}(x)$, we employ stationary log-normal random fields whose spatial correlation is represented by the auto-correlation function as follows:

$\rho_{m^{i}}\left(x_{1}, x_{2}\right)=\exp \left(-\frac{\left|x_{1}-x_{2}\right|}{l_{m^{i}}}\right)$,

where $x_{1}$ and $x_{2}$ are two points in a background domain, which is larger than all fracture length realizations, and $l_{m^{i}}$ is the correlation length. To generate samples of the random field, $\tilde{m}^{i}(x)$, it must be discretized. To obtain a discretization, the log-normal random field is considered as the exponential of an underlying stationary normal random field $\tilde{g}^{i}$ :

$\tilde{m}^{i}(x)=\exp \left(\tilde{g}^{i}(x)\right)$

The statistical moments of the underlying Gaussian distribution can be expressed in terms of those of the random field $\tilde{m}^{i}(x)$ by (see, e.g., [18])

$\mu_{g^{i}}=\ln \left(\mu_{m^{i}}\right)-\frac{1}{2} \ln \left(1+V_{m^{i}}^{2}\right), V_{g^{i}}=\sqrt{\ln \left(1+V_{m^{i}}^{2}\right)}$, where $V_{m^{i}}=\sigma_{m^{i}} / \mu_{m^{i}}$ is the coefficient of variation of the model parameter $m^{i}$. Similarly, the auto-correlation function can be written as follows:

$\rho_{g^{i}}\left(x_{1}, x_{2}\right)=\frac{\ln \left(1+\rho_{m^{i}}\left(x_{1}, x_{2}\right) V_{m^{i}}^{2}\right)}{\ln \left(1+V_{m^{i}}^{2}\right)}$.

Discretization of the underlying Gaussian random field $\tilde{g}^{i}(x)$ is then achieved by means of the Karhunen-Loève expansion (see, e.g., [38]) as follows:

$\tilde{g}^{i}(x) \approx g(x, \tilde{z})=\mu_{g^{i}}+\sum_{j=1}^{n} \sqrt{\xi_{j}} r_{j}(x) \tilde{z}_{j}$,

where $\tilde{z}$ is a vector of $n$ independent standard normal random variables, and where $\xi_{j}$ and $r_{j}(x)$ are the eigenvalues and eigenfunctions corresponding to the spatial covariance function $\sigma_{g^{i}}^{2} \rho_{g^{i}}\left(x_{1}, x_{2}\right)$, respectively. We discretize the eigenfunctions in space by means of a uniform linear finite element discretization over the background domain, which results in a generalized eigenvalue problem that we solve using a direct eigenvalue solver (see, e.g., Ref. [13] for details). In Fig. 3a, the first 12 numerically determined eigenfunctions are shown for the auto-correlation function (28) with $l_{m^{i}}=10 \mathrm{~m}$. To illustrate the effectiveness of the Karhunen-Loève expansion, Fig. $3 b$ shows the auto-correlation function reconstructed from the KarhunenLoève expansion (blue dots), which conveys that for this selection of the correlation length, an excellent approximation of the auto-correlation function is attained using 12 modes.

The log-normal random field $\tilde{m}^{i}(x)$ is then obtained by back-substitution of Eq. 32 into the transformation (29):

$\tilde{m}^{i}(x) \approx \frac{\mu_{m^{i}}}{\sqrt{1+V_{m^{i}}^{2}}} \prod_{j=1}^{n} \exp \left(\sqrt{\xi_{j}} r_{j}(x) \tilde{z}_{j}\right)$.

Realizations of the random field $\tilde{m}^{i}(x)$ can now be generated by sampling a sequence of $n$ independent standard normal random variables.

\section{Numerical simulations}

In this section, we present numerical results based on the methodology presented above. In Section 5.1, we first validate our methodology in a deterministic setting by consideration of the benchmark results presented in the comparative study by Warpinski et al. [41]. In this section, we demonstrate the necessity to use a tip enrichment function and enforcement of volume conservation, and we study the influence of the mesh size and time step size on the numerical results. In Section 5.2, the sensitivity of the observables-in particular, the fracture length and aperture - to the uncertain model parameters is 
Fig. 3 a Karhunen-Loève modes, $r_{j}(x)$, and a single realization (solid black line) in accordance with the expansion (32). b Prescribed and approximated auto-correlation function $\rho_{m^{i}}\left(x_{1}, x_{2}\right)$ for a correlation length of $l_{m^{i}}=10 \mathrm{~m}$

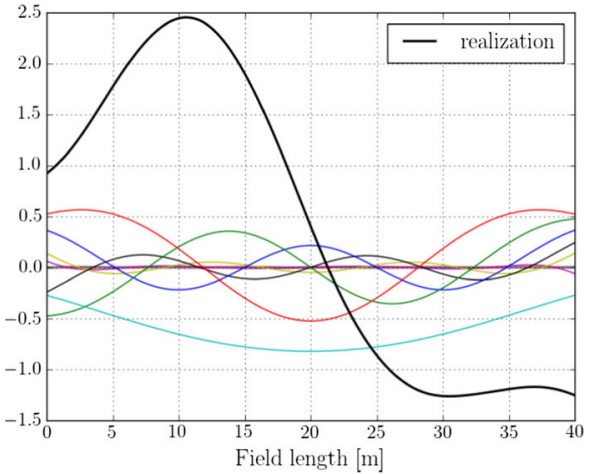

(a) Karhunen-Loève modes

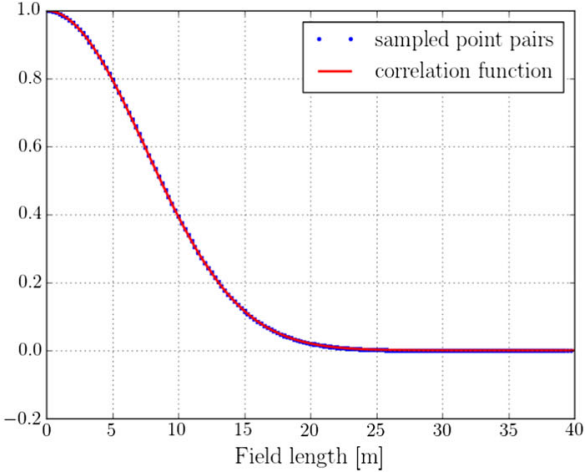

(b) Auto-correlation function studied, which serves as a starting point for the stochastic simulations discussed in Section 5.3. In the stochastic setting, the uncertain model parameters are represented by discrete random fields.

\subsection{Deterministic benchmark}

To demonstrate the validity of the presented methodology, we consider the benchmark case studied by Warpinski et al. [41], which is based on a staged-field experiment of the Gas Research Institute [41, p. 26]. The considered model parameters are assembled in Table 1 . The injection rate is gradually increased until the indicated value, and then held constant for $200 \mathrm{~min}$. The material parameters resemble that of a tight gas sand reservoir, for which spurt losses are omitted.

In Fig. 4, we show the evolution of the fracture in time, where it should be noted that the height of the fracture, $H$, is constant. Since the width is symmetric with respect to the $x$ axis, Fig. 4 displays half of the aperture. The shown results are based on a mesh size of $\Delta x=1 \mathrm{~m}$ and a time step size of $\Delta t=1 \mathrm{~s}$. As we will study in detail below, these results are objective with respect to these numerical parameters. Fig. 5 shows the corresponding increase in fracture length and fracture mouth opening over time. The observed time evolution corresponds well with the results reported for various simulators in [41] (displayed in Fig. 5 for reference). It is noted that the reported results in [41] vary

Table 1 Reservoir data used for the validation of the deterministic problem

\begin{tabular}{llll}
\hline Leak-off coefficient & $c_{l}$ & $9.84 \times 10^{-6}$ & $\mathrm{~m} / \mathrm{s}^{1 / 2}$ \\
Spurt losses & $S_{p}$ & 0 & $\mathrm{~m}$ \\
Fracture height & $H$ & 51.8 & $\mathrm{~m}$ \\
Plane strain modulus & $E^{\prime}$ & $6.13 \times 10^{10}$ & $\mathrm{~Pa}$ \\
Viscosity & $\mu_{f}$ & 0.2 & $\mathrm{~Pa} . \mathrm{s}$ \\
Injection rate & $i$ & 0.0662 & $\mathrm{~m}^{3} / \mathrm{s}$ \\
Pumping time & $T$ & 12000 & $\mathrm{~s}$ \\
\hline
\end{tabular}

significantly as a result of variations in model assumptions and simulation frameworks. The fracture length of $1429 \mathrm{~m}$ as computed here also corresponds reasonably well with the analytical model in [39], which-using additional simplifying assumptions-predicts a fracture length of $1340 \mathrm{~m}$. Note that in the absence of leak-off our model predicts a fracture length of $1730 \mathrm{~m}$. This stipulates that leak-off is appropriately represented in our numerical simulations. The fracture length and fracture opening computed by our methodology are in the higher part of the spectrum of simulators considered in [41] and analytical models, which we attribute to the explicit enforcement of the volume constraint, which will be discussed in detail below.

Our benchmark results are based on the formulation including the enrichment of the tip functions and the enforcement of the global volume conservation constraint (see Section 3). The results in Figs. 6 and 7 serve to illustrate that both these aspects are essential to obtain numerical results with an acceptable level of accuracy for meshes and time step sizes that are computationally tractable in the scope of stochastic simulations.

Figure 6 displays the behavior of the global volume conservation without and with Lagrange multiplier constraint. The total volume rate-which is the sum of the leak-off rate and fracture-widening rate-should equate to the input flow rate. Note that in the absence of the Lagrange multiplier constraint, a significant mismatch between the total rate and the inflow rate is observed. The presented figure is based on a mesh size of $\Delta x=1 \mathrm{~m}$ and a time step size of $\Delta t=1 \mathrm{~s}$. The mismatch between the rates depends on these discretization parameters, as it originates from significant errors in the local volume balance in the finite element discretization $(17 \mathrm{a}-\mathrm{d})$. These local inaccuracies in the finite element solution are closely related to the highly nonlinear character of the constitutive relation. By enforcing global conservation of volume using a Lagrange multiplier-as shown in Fig. 6a-the global loss of volume is rigorously resolved. As observed, the total volume rate in this case matches that of the inflow rate. 
Fig. 4 Aperture profiles evolving in time for the PKN benchmark simulation

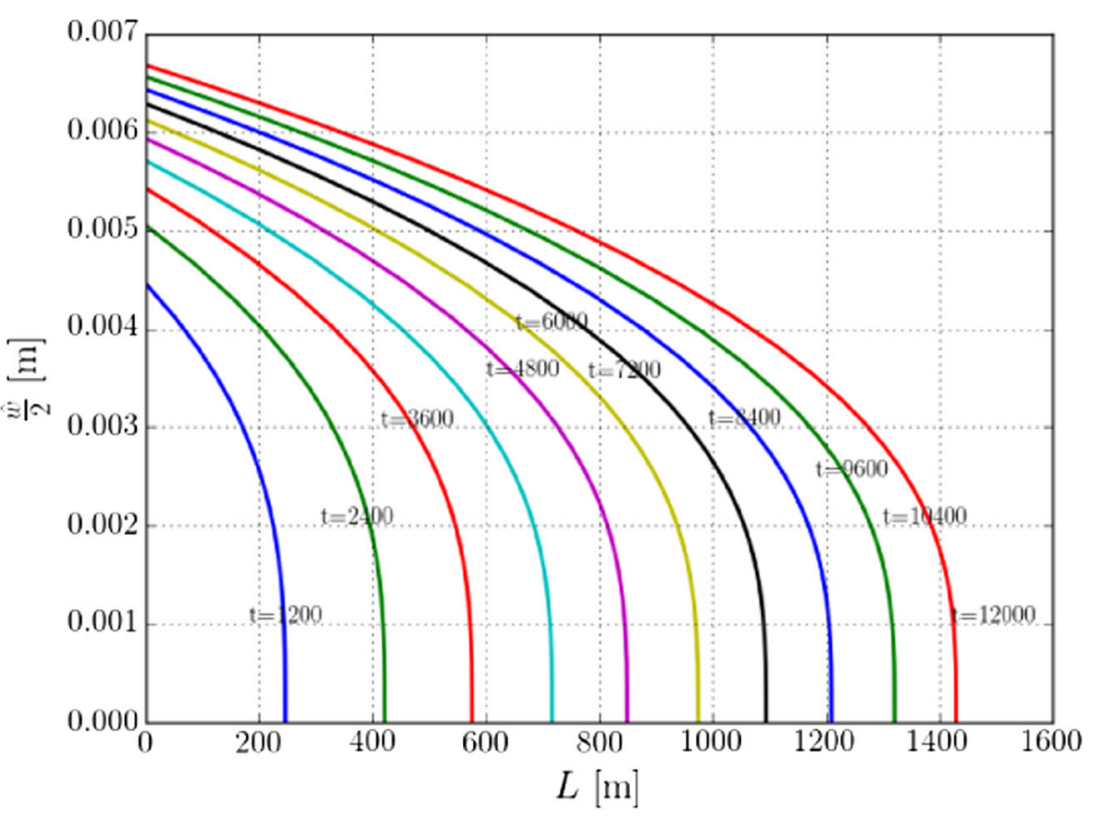

In Fig. 7, we display the tip velocity over time, without and with tip enrichment function. For both simulations, a mesh size of $\Delta x=1 \mathrm{~m}$ and a time step size of $\Delta t=1 \mathrm{~s}$ is used. In the case of tip enrichment, Eq. 14 is used to compute the tip speed. In the absence of enrichment, the tip speed cannot be obtained by this equation, as the adequate singular tip behavior is then not represented in the discrete solution space. The speed results presented in Fig. $7 \mathrm{~b}$ are based on the finite difference approximation as follows:

$\dot{L}(t)=-\frac{E^{\prime}}{96 \mu_{f} H}\left(\frac{\left.\hat{w}^{3}\right|_{L(t)}-\left.\hat{w}^{3}\right|_{(L(t)-\Delta x)}}{\Delta x}\right)$

From Fig. 7, it is observed that without the use of the enrichment function, for the computational setting considered here significant oscillations in the fracture propagation speed occur. Enriching the solution space with the tip singularity dramatically reduces these oscillations, and hence significantly improves the quality of the obtained result.

The enforcement of the volume constraint by means of a Lagrange multiplier, and the representation of the tip behavior by means of an enrichment function, provide numerical approximations with a level of accuracy that enables studying stochastic variations. In Figs. 8 and 9, we show the dependence of the results on independent variations in the time step size and mesh size, respectively. Note that in all simulations, we consider a duration of $50 \mathrm{~s}$ only, in order to make the converge studies feasible in terms of computational effort.

Figure 8 displays the results for a mesh size of $\Delta x=1 \mathrm{~m}$ using three time step sizes, viz. $\Delta t=1.0,0.5,0.25 \mathrm{~s}$. From both the length evolution plot and the tip speed evolution plot, it is observed that the variations with the time step size are very limited. The most notable difference is observed at the onset of fracture, where the maximum tip speed for $\Delta t=0.25 \mathrm{~s}$ is observed to be $2 \%$ higher than that for $\Delta t=1.0 \mathrm{~s}$. This difference is significantly smaller once steady propagation occurs, e.g. at $t=50 \mathrm{~s}$, where the difference is only $0.8 \%$. Since the length of the fracture is generally not dominated by the onset phase, the observed variation in fracture length is generally also very small. At $t=50 \mathrm{~s}$, the fracture length for $\Delta t=1.0$ underestimates that of $\Delta t=0.25$ by $0.5 \%$. Although not presented here for the sake of brevity, similar results can be established for other indicators such as the fracture mouth opening.

The evolution of the fracture length and tip speed for $\Delta t=0.5 s$ and $\Delta x=0.5,1,2 \mathrm{~m}$ are depicted in Fig. 9 . The figures show that although a mesh size of $\Delta x=2.0 \mathrm{~m}$ correctly mimics the tip speed behavior, fluctuations in the speed can be observed as a consequence of the mesh coarseness. These fluctuations can be attributed to the fact that due to the limited number of elements in this simulation (i.e., only 10 elements at $t=50 \mathrm{~s}$ ), the spatial discretization errors resulting from the employed moving-mesh approach are significant. Upon mesh refinement, these fluctuations vanish. The maximum tip speed at the onset of fracture for a mesh with $\Delta x=0.5 \mathrm{~m}$ is only $0.5 \%$ lower than that using a twice coarser mesh. When the fracture is steady at $t=50 \mathrm{~s}$, this relative difference is even smaller. In terms of the fracture length at $t=50 \mathrm{~s}$, the result for $\Delta x=1.0 \mathrm{~m}$ underestimates that for $\Delta x=0.5 \mathrm{~m}$ by a mere $0.5 \%$.

In the context of the sensitivity studies and stochastic simulations considered in the remainder of this section, it is essential that the numerical errors do not pollute the results. 
Fig. 5 Evolution of a the fracture length and $\mathbf{b}$ the maximum aperture over time as computed by the finite element methodology proposed in this contribution (solid blue line).

The benchmark results reported in Ref. [41] are displayed for references. See Appendix for further details

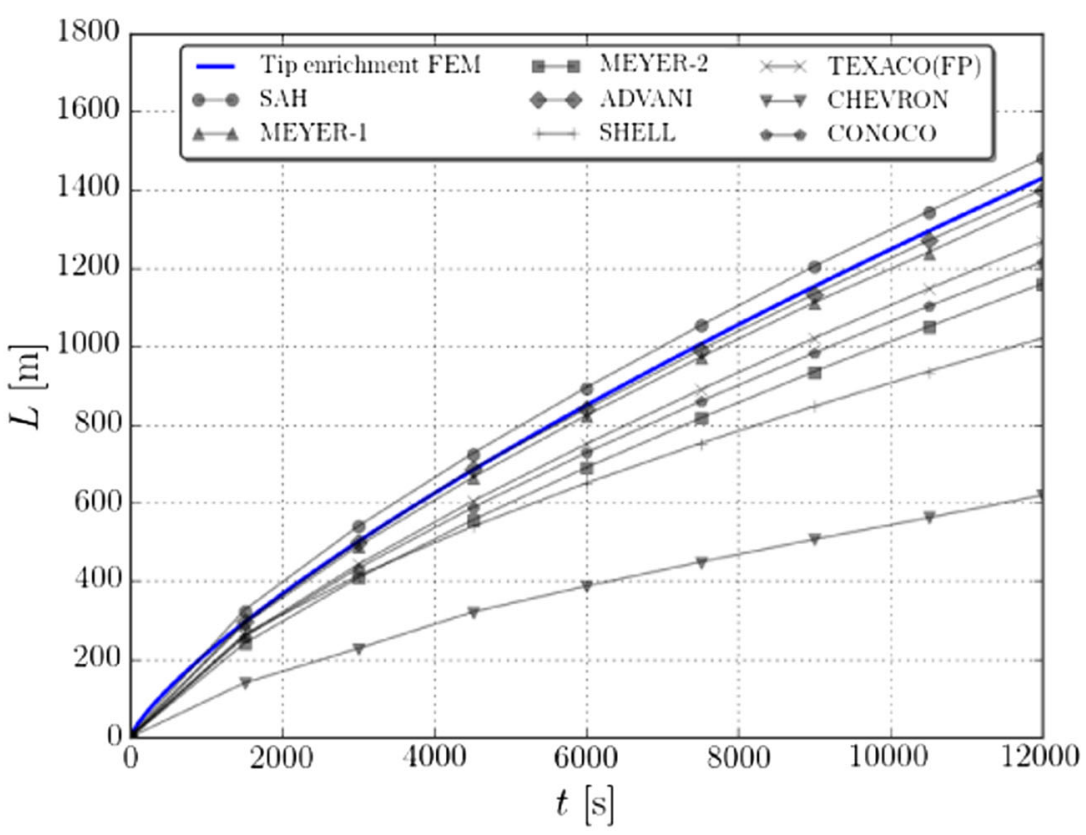

(a)

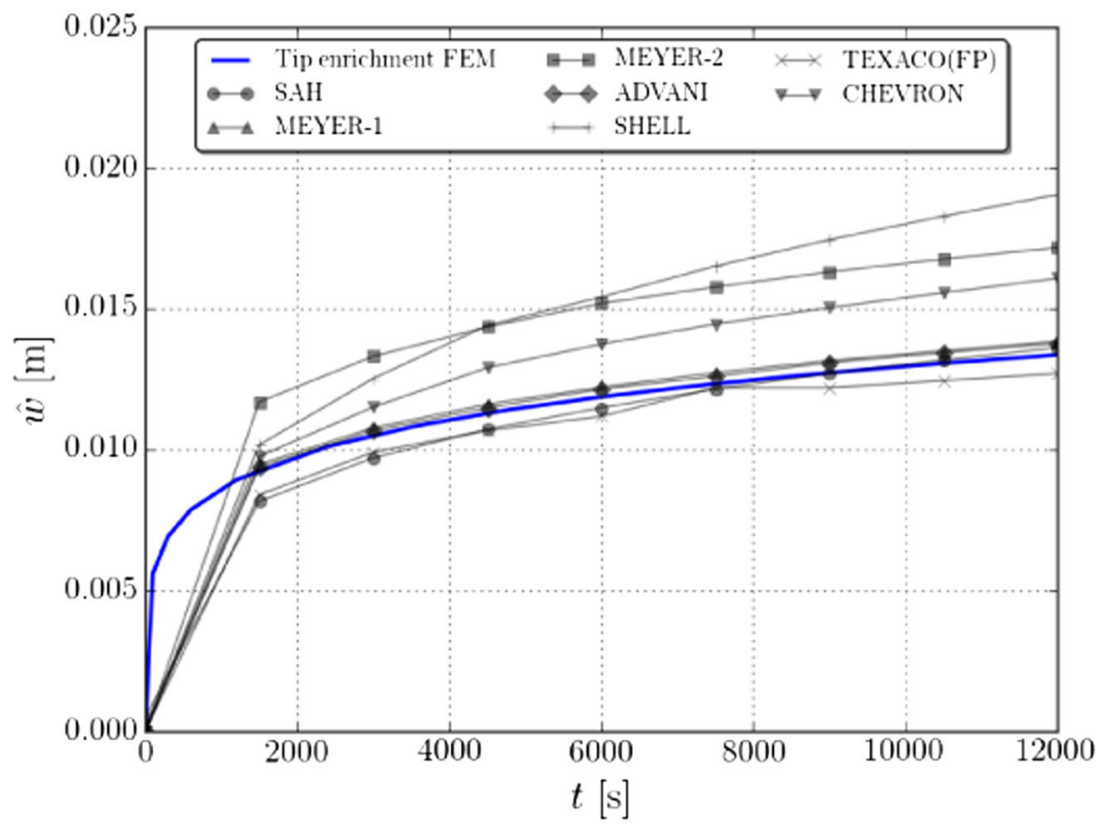

(b)

This means that the variations discussed above should be negligible in comparison to the stochastic variations in the input parameters. On the other hand, making the mesh sizes and time step sizes too small will dramatically increase the computational effort due to the large number of samples that is required in accordance with Eq. 27. Herein, we select a mesh size of $\Delta x=1.0 \mathrm{~m}$ and a time step size $\Delta t=1.0 \mathrm{~s}$, which provides a good balance between numerical accuracy (see above) and computational effort.

\subsection{Sensitivity analysis}

In this section, we apply the deterministic model outlined above to identify the input factors that drive the variation in the output. As output observables, we consider the fracture length and fracture aperture. As input parameters, we consider the model parameters that cannot be established with a high degree of certainty in reality, viz. the plane strain modulus $E^{\prime}$, the fracture height, $H$, and the leak-off coefficient, $c_{l}$. In our sensitivity analysis, we independently 
Fig. 6 Influence of the Lagrange multiplier on the conservation of volume

Fig. 7 Influence of the enrichment function on the tip propagation velocity

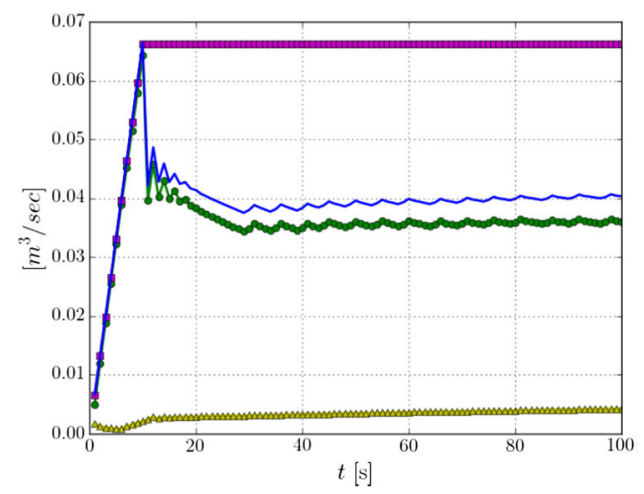

(a) With Lagrange multiplier

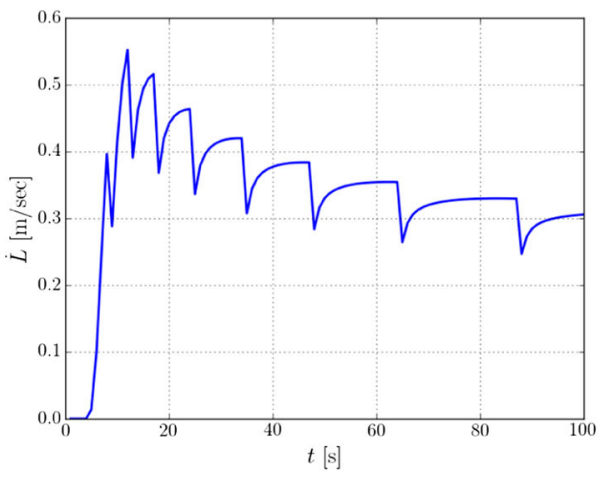

(a) With enrichment

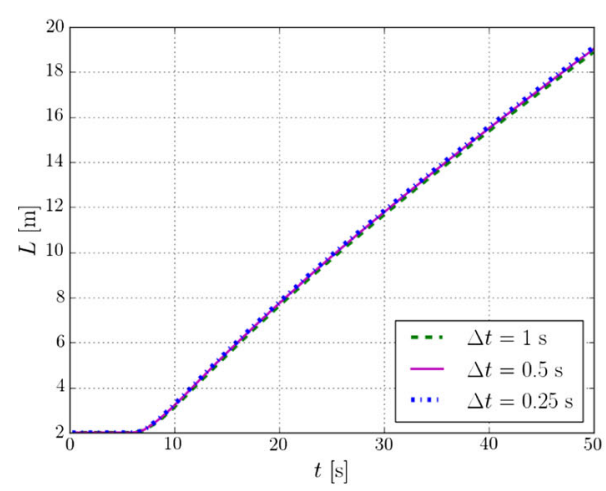

(a)

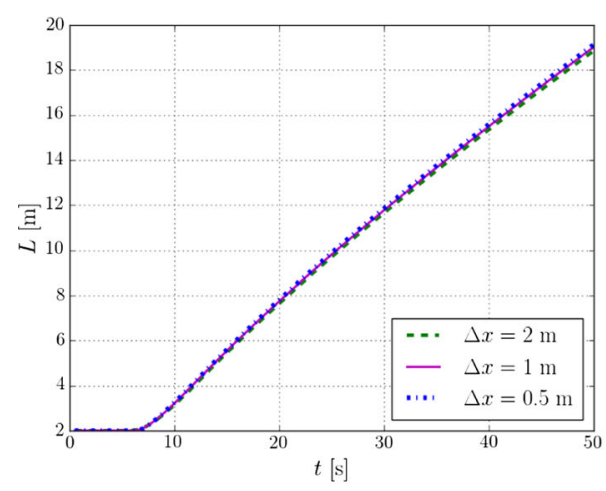

(a)

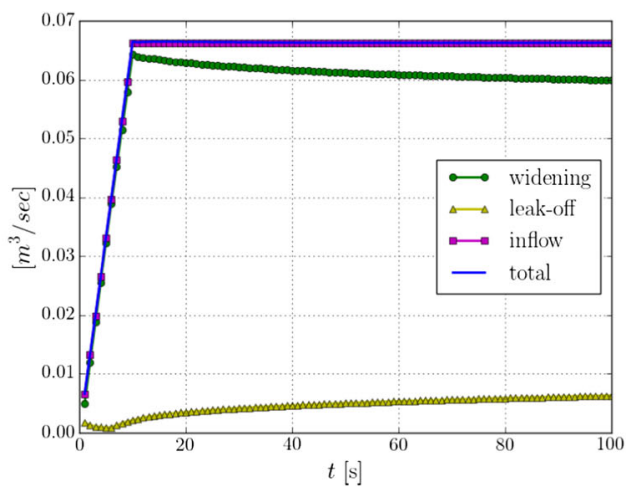

(b) Without Lagrange multiplier

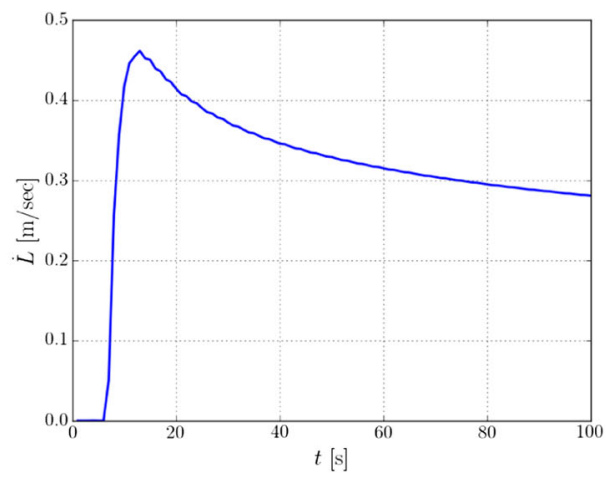

(b) Without enrichment

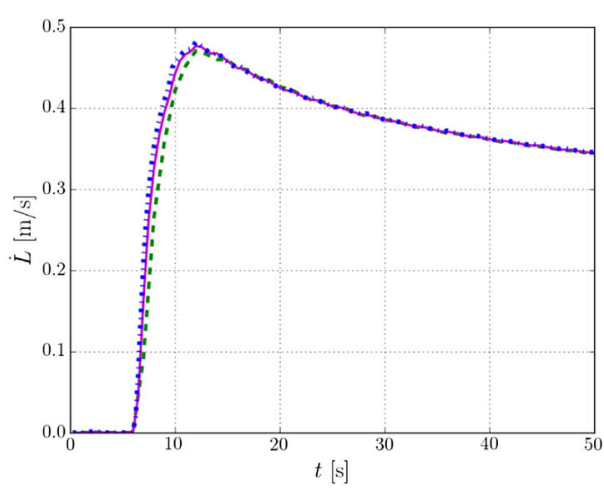

(b)

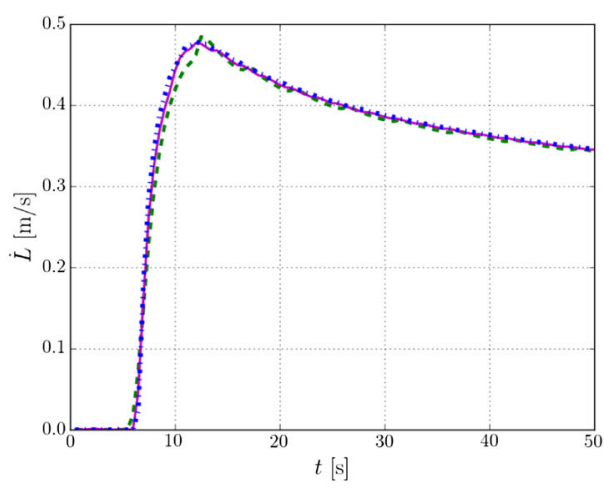

(b) 
Fig. 10 a Fracture length and aperture with varying plane strain modulus and $\mathbf{b}$ fracture geometry profile with varying plane strain modulus

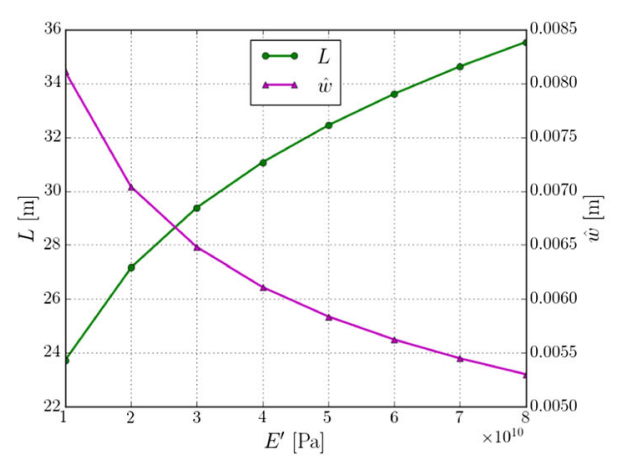

(a)

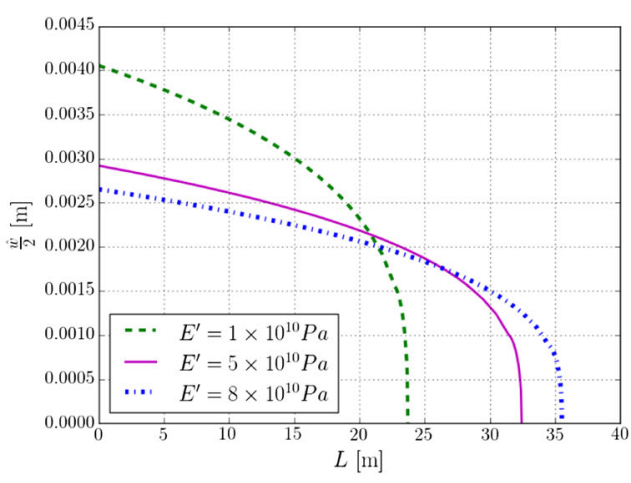

(b) vary the input factors and study their impact on the output observables. This screening procedure is often considered as the first step in a forward uncertainty analysis, since it identifies the dominant sources of randomness.

In Fig. 10, we consider the effect of the plane strain modulus on the fracture geometry, while keeping all other model parameters unchanged. A range of plane strain moduli from $1 \times 10^{3}$ to $1 \times 10^{4} \mathrm{MPa}$ is considered. Figure 10 conveys that a stiffer formation results in a longer and narrower fracture, compared to a more compliant formation. Considering the nature of the model-which revolves around the conservation of volume-this is plausible, since in the case of a stiff formation fracture propagation is favored over fracture widening. From Fig. 10a, we observe that the dependence of the output observables on the plane strain modulus is highly nonlinear, in the sense that the rate of change of the output observables is significantly lower than that of the plane strain modulus. For example, an increase of $45 \%$ in the fracture length is observed when increasing the plane strain modulus by a factor of 8 . This same increase in plane strain modulus, moreover leads to a reduction of the fracture mouth opening by only $35 \%$. As a result, the well pressure-which is proportional to the product of the fracture mouth opening and plane strain modulus - increases with an increase in formation stiffness, which is in line with the experimental results in [41]. In addition, this sensitivity analysis conveys that the response of the observables is non-symmetric, in the sense that the rate of change of the length and fracture mouth opening for stiff formations is smaller than for compliant formations.

Figure 11 shows the dependence of the output observables on a range of leak-off coefficients, ranging from the impermeable case $\left(c_{l}=0 \mathrm{~m} / \mathrm{s}^{1 / 2}\right)$ to $c_{l}=5 e^{-5} \mathrm{~m} / \mathrm{s}^{1 / 2}$, which is $50 \%$ more than the value taken in the GRI experiment [41] based on tight gas sands. As anticipated from the conservation of volume, an increasing leak-off coefficient yields a shorter and narrower crack. Increasing the deterministic value considered in the previous section by a factor of 5 yields a decrease in fracture length of $14 \%$ and a decrease in fracture mouth opening of $7 \%$. From Fig. 11b, it is observed that the fracture profile shape is insensitive to the leak-off coefficient. In contrast to the dependence on the plane strain modulus considered above, the rate of change of the observables is practically constant for the considered range of leak-off coefficients.

Figure 12 displays the variation of the output observables for fracture heights ranging from 25 to $95 \mathrm{~m}$. Doubling the fracture height from $25 \mathrm{~m}$ reduces the fracture mouth
Fig. 11 a Fracture length and aperture with varying leak-off coefficient and $\mathbf{b}$ fracture geometry profile with varying leak-off coefficient

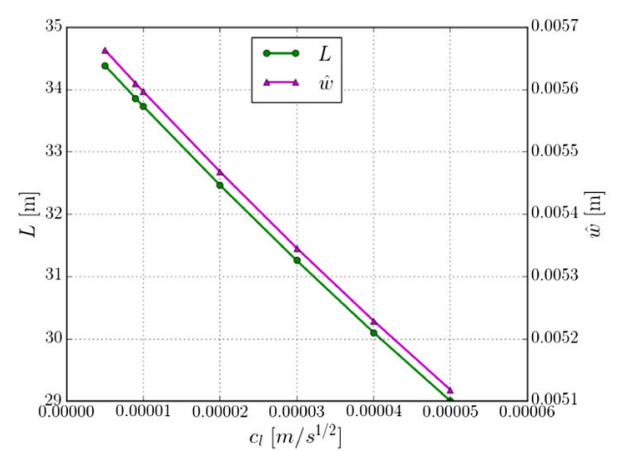

(a)

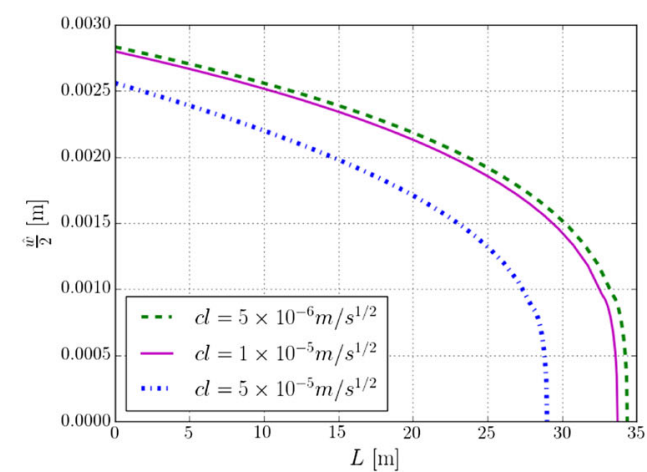

(b) 
Fig. 12 a Fracture length and aperture with varying fracture height, and $\mathbf{b}$ fracture geometry profile with varying fracture height

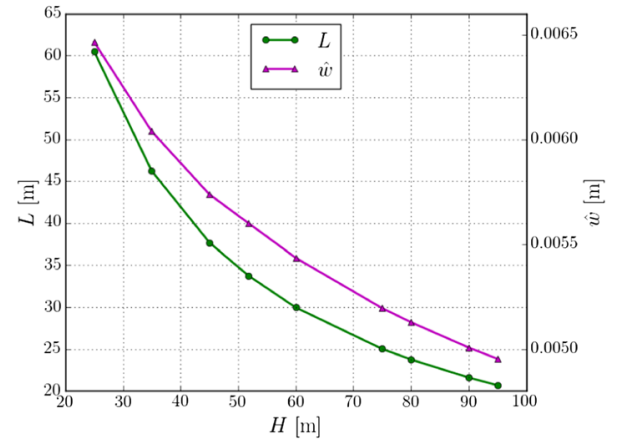

(a)

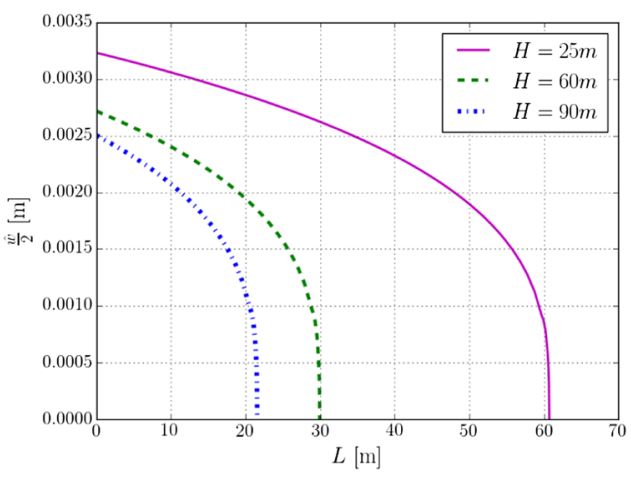

(b) opening by $15 \%$ and the fracture length by $44 \%$. As expected from volume conservation (where in this particular case leak-off effects are not pronounced), the product of these two observables is approximately reduced by a factor of two. The direct impact of the fracture height on the volume conservation model results in a strong sensitivity of the output observables. The non-symmetry of the response observables is consistent with the expected behavior in the extreme cases, for which a large height should yield a very short and narrow crack, and a small height should yield an extremely long and wide fracture.

\subsection{Stochastic setting}

In this section, we present the results of Monte-Carlo simulations. In Section 5.3.1, we first study the stochastic results for the case where each of the uncertain input

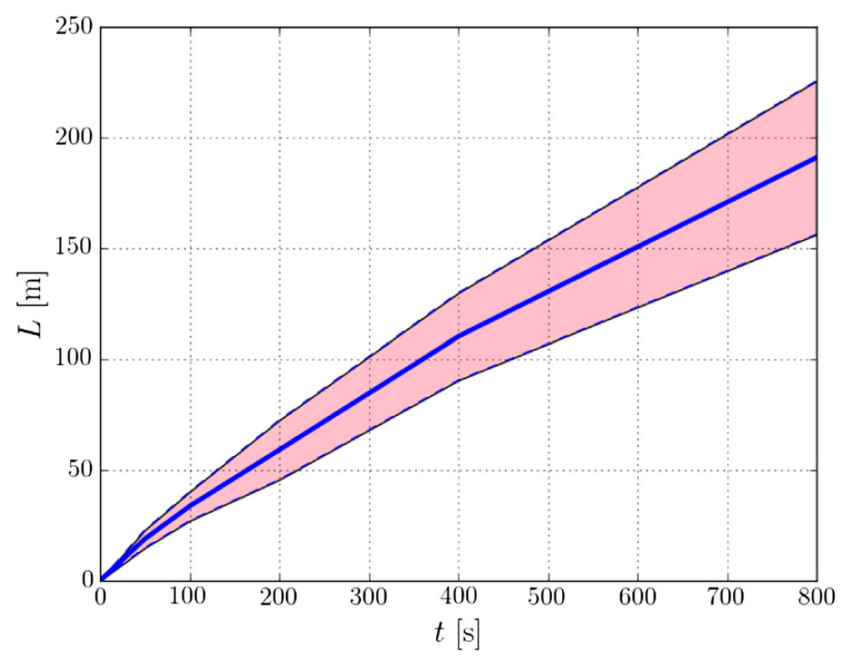

Fig. 13 Evolution of the mean (solid blue line) and 98\% confidence interval (shaded area) of the fracture length in time corresponding to a variation in the plane strain modulus of $V_{E^{\prime}}=50 \%$ parameters is varied independently, which closely connects this section to the sensitivity analysis presented above. The stochastic analysis presented here provides insight into the evolution of the randomness in time, and on the dependence of the uncertain observables on the magnitude of the random input variables. In Section 5.3.2, we then consider the case of a random field for the plane strain modulus, which elucidates the dependence of the observables on the spatial correlation of the uncertain input parameter. The reported sample sizes are all based on the estimate (27) with a confidence level for the estimator of the mean fracture length.

\subsubsection{Independent variation of uncertain parameters}

We first consider the plane strain modulus, $E^{\prime}$, to be the only uncertain parameter. Since the plane strain modulus is positive by definition, we represent this uncertain parameter by a log-normal distribution with mean value $\mu_{E^{\prime}}=6.13 \times$ $10^{4} \mathrm{MPa}$ and coefficient of variation $V_{E^{\prime}}=50 \%$. To achieve a confidence level of $98 \%$ for the mean fracture length, a sample size of $N=304$ is required. To avoid pollution of the stochastic results by excessive discretization errors for this $98 \%$ confidence interval, a mesh size $\Delta x=1 \mathrm{~m}$ and a time step size $\Delta t=1 \mathrm{~s}$ is used in combination with the volume conservation constraint and tip enrichment function. In this computational setting, a single realization with $T=800 \mathrm{~s}$ requires $96 \mathrm{~min}$ on a Intel Core i5 vPro processor. Without the use of the volume constraint and enrichment function, significantly smaller mesh and time step sizes would be required to achieve the numerical accuracy required for a 98\% confidence interval, which would dramatically increase the total simulation time of the Monte-Carlo simulation.

In Fig. 13, we show the evolution of the fracture length in time, where the mean value is represented by the solid 
Fig. 14 Histograms of the fracture length and maximum fracture aperture for $50 \%$ variation of the plane strain modulus, $E^{\prime}$, at $t=100 \mathrm{~s}$

Table 2 Statistical moments of the fracture length and fracture aperture corresponding to a coefficient of variation $V_{E^{\prime}}=50 \%$ for the plane strain modulus

Fig. 15 Coefficients of variation of the fracture length, $L$, and maximum aperture, $\hat{w}$, as a function of the variation of the plane strain modulus, $E^{\prime}$, at different time instances

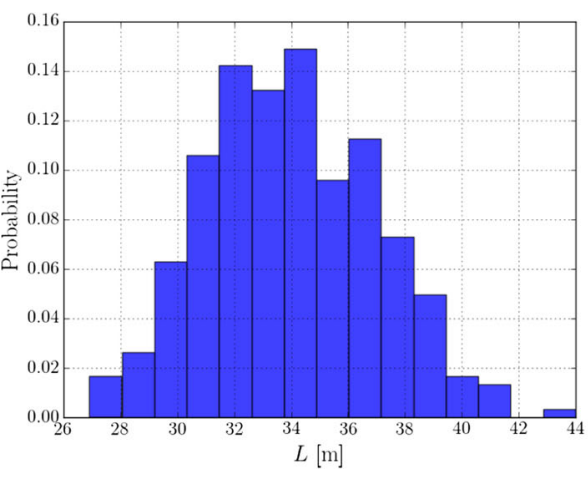

(a) Fracture length, $L$

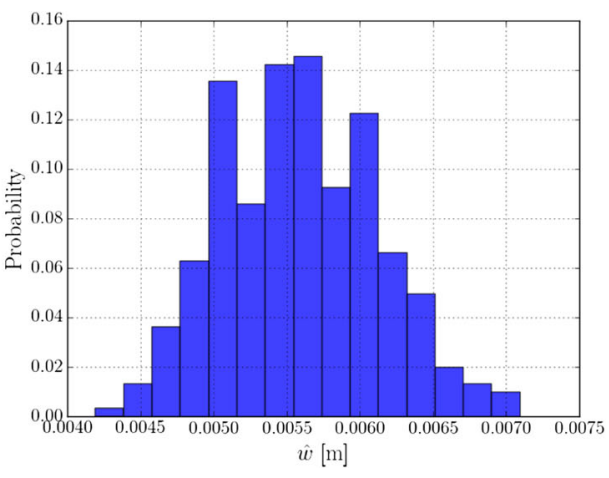

(b) Maximum aperture, $\hat{w}$

\begin{tabular}{lllllll}
\hline$t$ & $\mu_{L}$ & $\sigma_{L}$ & $V_{L}$ & $\mu_{\hat{w}}$ & $\sigma_{\hat{w}}$ & $V_{\hat{w}}$ \\
\hline 100 & 33.619 & 3.348 & 9.9 & 0.0056 & 0.0005 & 8.9 \\
200 & 59.040 & 5.790 & 9.8 & 0.0066 & 0.0006 & 9.1 \\
400 & 110.166 & 10.030 & 9.1 & 0.0076 & 0.0007 & 9.2 \\
\hline
\end{tabular}

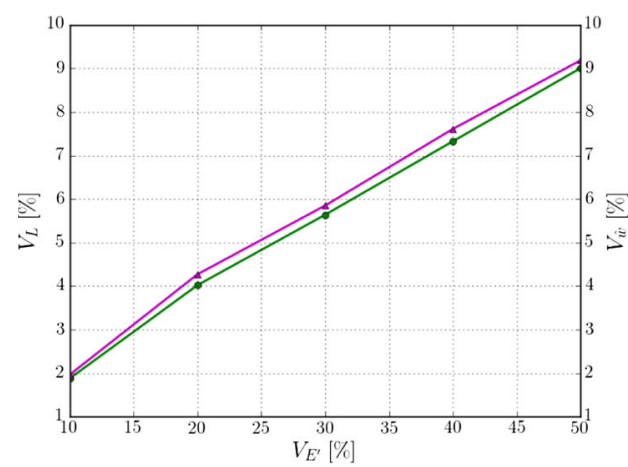

(a) $t=100 \mathrm{~s}$

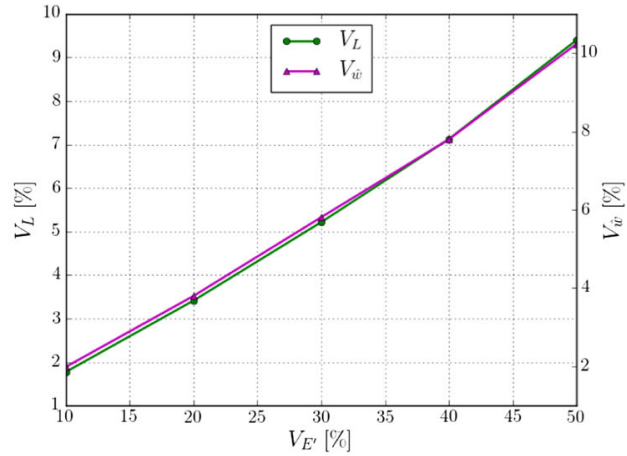

(b) $t=500 \mathrm{~s}$
Fig. 16 Statistical moments of the fracture length, $L$, and maximum aperture, $\hat{w}$, as a function of the variation of the plane strain modulus, $E^{\prime}$, at $t=100 \mathrm{~s}$. The perturbation results, $L^{p}$ and $\hat{w}^{p}$, are indicated by the dashed lines

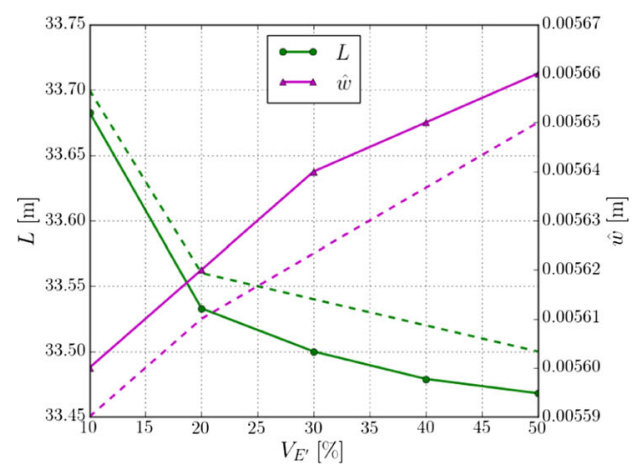

(a) Mean

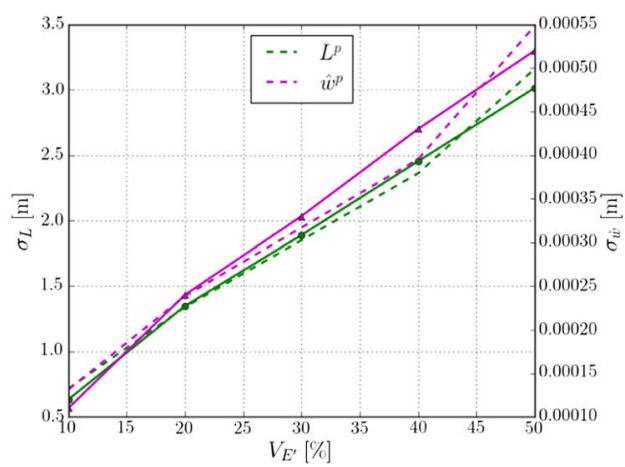

(b) Standard deviation 


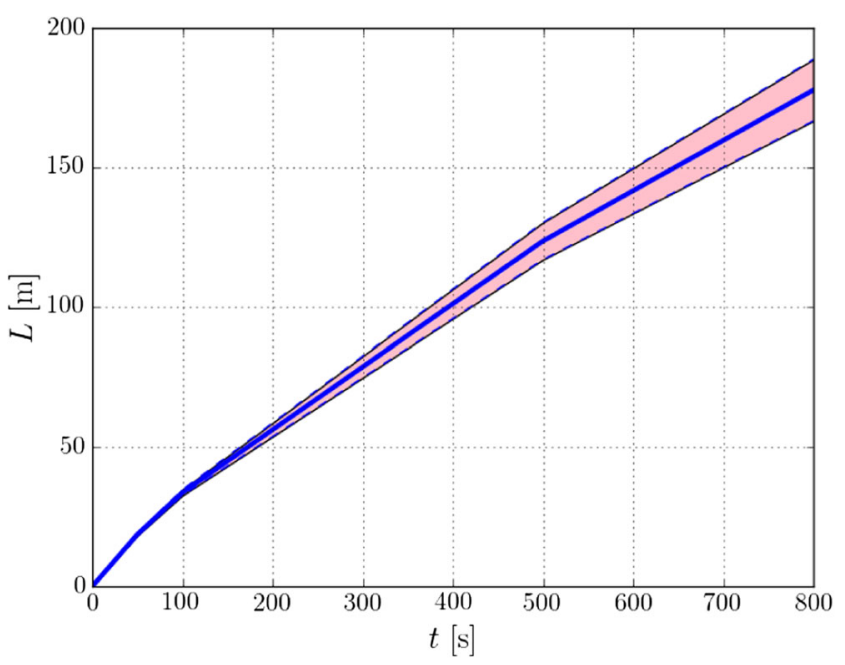

Fig. 17 Evolution of the mean (solid blue line) and 98\% confidence interval (shaded area) of the fracture length in time corresponding to a variation in the leak-off coefficient of $V_{c l}=50 \%$

line, and the shaded area indicates the $98 \%$ confidence interval of the fracture length. Typical distributions for the fracture length and fracture mouth opening at $t=100 \mathrm{~s}$ are displayed in Fig. 14, from which we infer that the distributions are unimodal. The error bars in Fig. 13 show that the standard deviation of the fracture length (and with that its confidence interval) increases proportionally with the mean. This observation is confirmed in Table 2, from which it is observed that the coefficients of variation of the length and fracture mouth opening only vary moderately.

In Fig. 15, we study the dependence of the results on the coefficient of variation of the plane strain modulus at two time instances. Note that the used sample size is different for each coefficient of variation in order to achieve the same confidence level for the mean estimator of the fracture length. At both time instances, we observe that the coefficient of variation of the fracture length is proportional to that of the input parameter. This behavior can be explained by considering the dependence of the mean and the standard deviation on the coefficient of variation of the input, as displayed in Fig. 16. This figure conveys that the standard deviation of the fracture length increases with an increasing variation of the plane strain modulus. Using the results from the sensitivity analysis presented above, we verify the correspondence of the observed behavior with that predicted by a first-order perturbation analysis [13],

$\sigma_{L} \approx\left|\frac{\partial L}{\partial E^{\prime}}\right|_{E^{\prime}=\mu_{E^{\prime}}} \frac{\partial E}{\partial z} \approx\left|\frac{\partial L}{\partial E^{\prime}}\right|_{E^{\prime}=\mu_{E^{\prime}}} \sigma_{E^{\prime}}$,

which is displayed in Fig. 16b by the dashed lines. The observed decrease in the mean value is also in good agreement with the results of the sensitivity analysis, as shown by the dashed lines in the Fig. 16a, where the mean values are approximated using second-order perturbation theory [13]:

$\left.\mu_{L} \approx L\right|_{E^{\prime}=\mu_{E^{\prime}}}+\frac{1}{2} \frac{\partial^{2} L}{\partial E^{\prime 2}} \sigma_{E^{\prime}}^{2}$

For the case considered here, the variation in the mean of the fracture length is moderate (Fig. 16a), as a result of which the observed behavior of the coefficient of variation in Fig. 15 is governed by the behavior of the standard deviation of the fracture length. Similarly, the results for the fracture mouth opening are also observed to correspond well with the sensitivity analysis.

In Fig. 17, we show the evolution of the fracture length for the case in which the leak-off coefficient is described by a log-normal distribution with mean value $\mu_{c_{l}}=9.84 \times$ $10^{-6} \mathrm{~m} / \mathrm{s}^{1 / 2}$ and coefficient of variation $V_{c_{l}}=50 \%$. The probability distributions at $t=100 \mathrm{~s}$ are shown in Fig. 18. Figure 19 presents the corresponding relation between the coefficients of variation of the observables, viz. the fracture length and maximum aperture, and that of the leak-off coefficient at two time instances, where the sample sizes have been selected in accordance with
Fig. 18 Histograms of the fracture length and maximum fracture aperture for $50 \%$ variation of the leak-off coefficient, $c_{l}$, at $t=100 \mathrm{~s}$

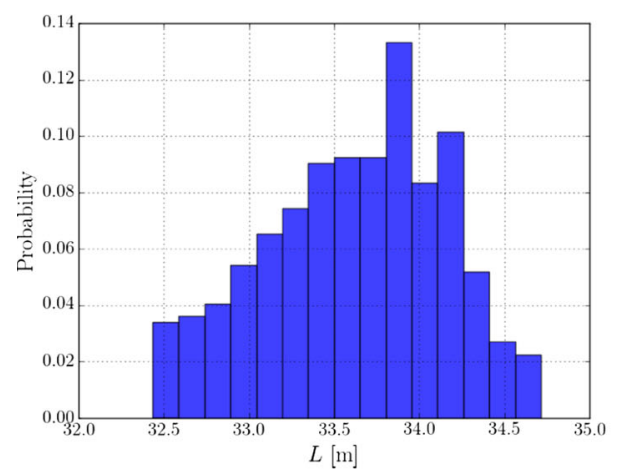

(a) Fracture length, $L$

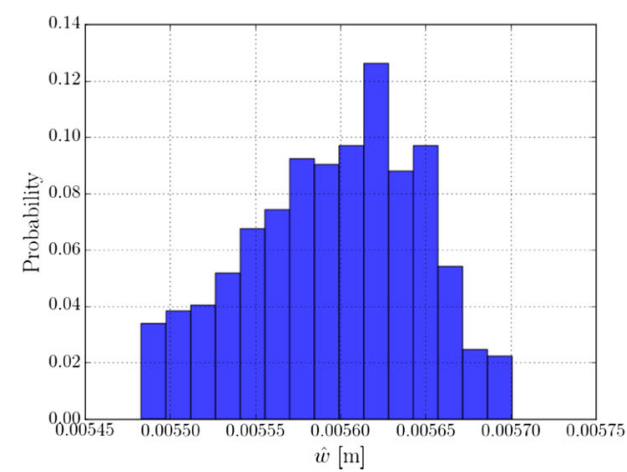

(b) Maximum aperture, $\hat{w}$ 
Fig. 19 Coefficients of variation of the fracture length, $L$, and maximum aperture, $\hat{w}$, as a function of the variation of the leak-off coefficient, $c_{l}$, at different time instances

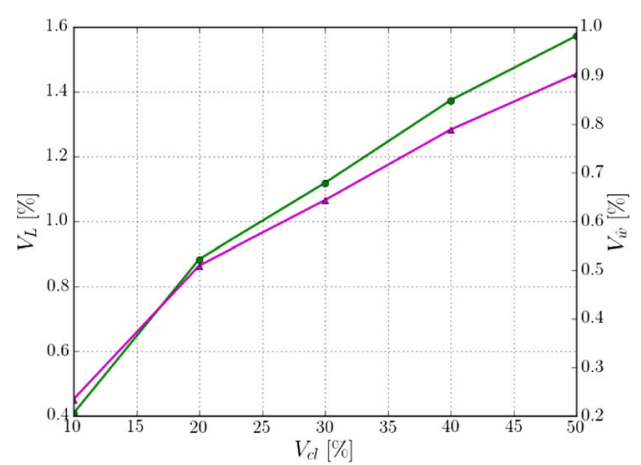

(a) $t=100 \mathrm{~s}$

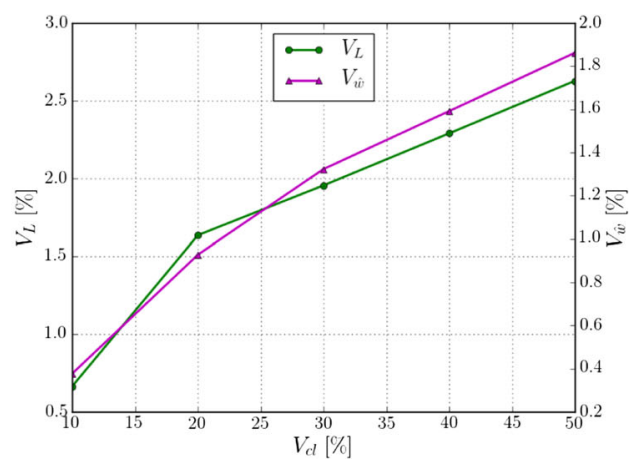

(b) $t=500 \mathrm{~s}$ the confidence level of the mean estimator of the fracture length. From Fig. 19, one can observe that the coefficients of variation of the output observables increase only modestly (by approximately a factor of 2 from $t=100 \mathrm{~s}$ to $t=500 \mathrm{~s}$ ) in time. The observed relation between the coefficients of variation of the input and output is explained by the fact that the mean value is affected minimally by the coefficient of variation of the leak-off coefficient, while the standard deviation increases proportionally with it. From Fig. 20, it is observed that the behavior of the mean and standard deviation of the observables is in good agreement with the perturbation results following from the sensitivity analysis.

We finally consider the independent random variation of the fracture height, which is represented by a log-normal distribution with mean $\mu_{H}=51.8 \mathrm{~m}$ and coefficient of variation $V_{H}=50 \%$. A sample size of $N=553$ (providing a confidence level of $95 \%$ ) is selected to compute the time evolution of the fracture length as shown in Fig. 21, for which the probability distributions of fracture length $L$ and maximum aperture $\hat{w}$ at time $t=100 \mathrm{~s}$ are shown in Fig. 22. The coefficient of variation is essentially invariant in time, which is confirmed by comparison of the relation between the input and output coefficients of variation at two time instances as shown in Fig. 23. From Fig. 24, we observe that both the mean and the standard deviation of the observables match well with the results from the sensitivity analysis.

Comparing the effects of the random input variable on the fracture length reveals that it is more sensitive to randomness in the plane strain modulus (Fig. 15) than to randomness in the leak-off coefficient (Fig. 19), in the sense that a coefficient of variation in the fracture length of $V_{L} \approx 2.5 \%$ corresponds to a coefficient of variation of the plane strain modulus of $V_{E^{\prime}} \approx 15 \%$ and a coefficient of variation of the leak-off coefficient of $V_{c_{l}}=50 \%$ (results at $t=500 \mathrm{~s}$ ). The sensitivity to the fracture height is observed to be even stronger (Fig. 23), in the sense that $V_{L} \approx 10 \%$ corresponds to coefficients of variation of $V_{E^{\prime}} \approx 50 \%$ and $V_{H} \approx 15 \%$ for the plane strain modulus and fracture height, respectively.

\subsubsection{Heterogeneous random plane strain modulus field}

We now consider the case in which the plane strain modulus, $E^{\prime}$, is described by a heterogeneous random field instead of a scalar variable. We consider a random field with mean $\mu_{E^{\prime}}=6.13 \times 10^{3} \mathrm{MPa}$ and coefficient of variation
Fig. 20 Statistical moments of the fracture length, $L$, and maximum aperture, $\hat{w}$, as a function of the variation of the leak-off coefficient, $c_{l}$, at $t=100 \mathrm{~s}$. The perturbation results, $L^{p}$ and $\hat{w}^{p}$, are indicated by the dashed lines

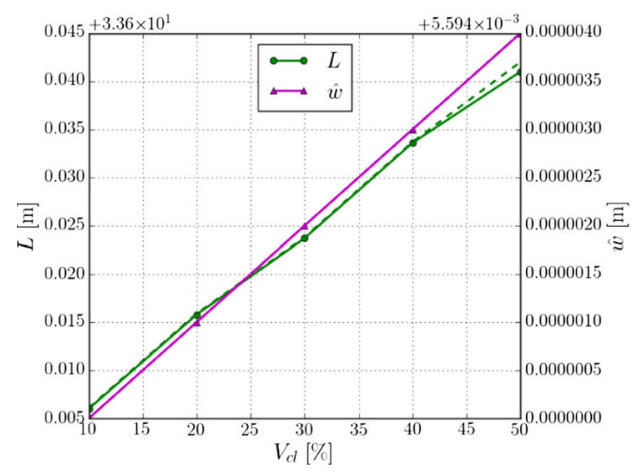

(a) Mean

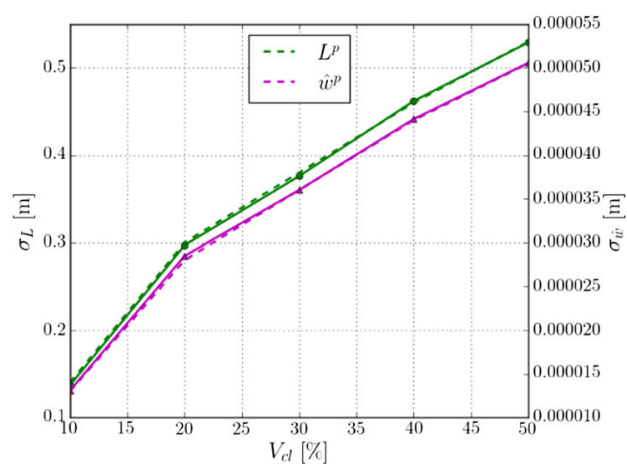

(b) Standard deviation 


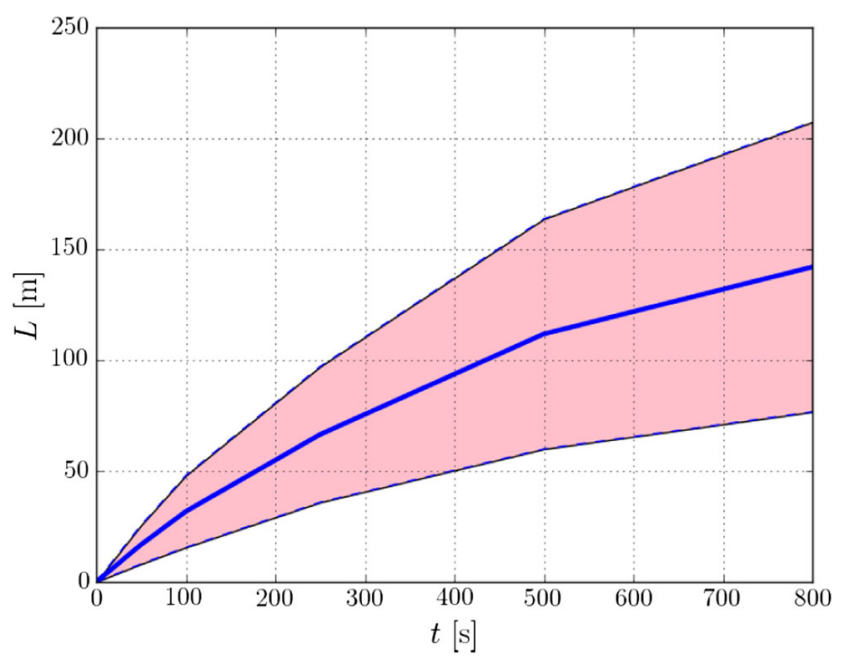

Fig. 21 Evolution of the mean (solid blue line) and 95\% confidence interval (shaded area) of the fracture length in time corresponding to a variation in the fracture height of $V_{H}=50 \%$

$V_{E^{\prime}}=50 \%$. We vary the length scale of the auto-correlation function in Eq. 31 from $l_{E^{\prime}}=5 \mathrm{~m}$ to $l_{E^{\prime}}=25 \mathrm{~m}$. The random field for the plane strain modulus is discretized using 12 Karhunen-Loève modes, which is sufficient for the representation of the random field corresponding to the smallest correlation length considered. Table 3 lists the statistical moments of the observables at $t=100 \mathrm{~s}$ based on a Monte-Carlo simulation with $N=384$, which is in accordance with a $95 \%$ confidence level for the mean estimator in the fracture length.

The most notable observation from the results in Table 3 is that the coefficient of variation of the output observables is significantly higher than in the case of a homogeneous plane strain modulus with equal coefficient of variation (see Table 2). To better understand this observation, in Fig. 25, we perform a closer inspection of the realizations that lead to Table 3 . In the rows of this figure, we collect the Monte-Carlo results for the correlation lengths reported in Table 3, starting with the smallest correlation length. In the second and third column, we show the probability distributions for the fracture length and the fracture mouth opening, respectively. The first column displays the plane strain modulus field that leads to three distinct realizations in the sample, viz. the smallest fracture length, the largest fracture length, and the fracture length closest to the mean value. We observe that the realizations of the plane strain modulus field that lead to the smallest fracture lengths in all cases correspond to the situation in which the elastic modulus is very small near the well. When this happens, the injected fluid causes fracture widening near the well, rather than fracture propagation into the formation. More generally, in the case of heterogeneous fields, local zones in which the formation is very compliant can lead to blockage of propagation, as the injected fluid volume can be locally accumulated in this zone. Long fractures are obtained in the case that the plane strain modulus is large near the well, and high (in a spatially averaged sense) compared to the mean value. In such situations, the blockage of propagation due to a compliant zone does not occur, and the injected fluid volume is effectively transferred to the crack tip.

In terms of the dependence of the results on the correlation length, it is observed that the mean fracture length decreases as the correlation length decreases. This is explained by the fact that in the case of a smaller correlation length, the chance of a locally compliant zone in the formation increases. The blockage of propagation in such zones is then more frequent, which leads to a reduction in fracture length expectation. From Table 3, we moreover observe a moderate increase in coefficient of variation of the fracture length as the correlation length increases.

From the distributions of the fracture mouth opening in Fig. 25c1-c5, we observe a notable difference in comparison to that for the homogeneous random plane strain modulus case (Fig. 14b). In the homogeneous case, there exists a strong correlation between the fracture length and the fracture mouth opening, in the sense that long cracks are narrow by virtue of the fact that their volume is similar (assuming leak-off effects to be limited). Figure 10 in the sensitivity study clearly confirms this observation; see also the discussion in Section 5.2. Although the fracture length and fracture width in the case of a heterogeneous field are not uncorrelated, the fracture mouth opening is most strongly influenced by the local plane strain modulus near the well. Since the fracture opening in the PKN model depends locally on the plane strain modulus, the log-normal distribution of the plane strain modulus reflects directly on that of the fracture mouth opening, as can be seen in the third column of Fig. 25. The sensitivity of the fracture mouth opening to local variations in the plane strain modulus field also results in coefficients of variation that are significantly higher than those in the homogeneous case (see Table 3 ).

\section{Conclusions}

We have presented a sampling-based stochastic analysis of the hydraulic fracturing process based on the PerkinsKern-Nordgren (PKN) model. The consideration of this model is motivated by the fact that in the deterministic case high-accuracy solutions can be computed with feasible computational effort, which makes its application in the context of direct Monte-Carlo sampling practical. Although this model significantly simplifies the hydraulic fracturing process, it bears practical relevance, especially for fractures in the viscosity-dominated regime. A limitation of the model 
Fig. 22 Histograms of the fracture length and maximum fracture aperture for $50 \%$ variation of the fracture height, $H$, at $t=100 \mathrm{~s}$

ig. 23 Coefficients of variation of the fracture length, $L$, and maximum aperture, $\hat{w}$, as a function of the variation of the time instances fracture height, $H$, at different

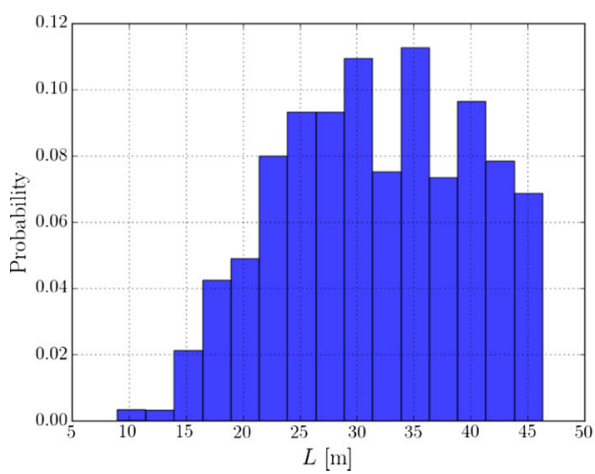

(a) Fracture length, $L$

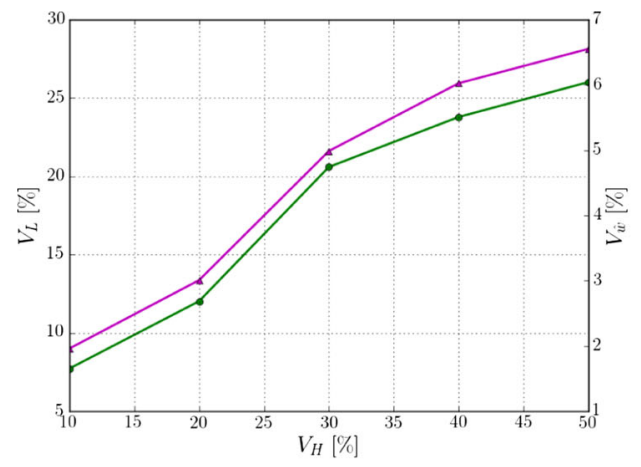

(a) $t=100 \mathrm{~s}$

Fig. 24 Statistical moments of the fracture length, $L$, and maximum aperture, $\hat{w}$, as a function of the variation of the fracture height, $H$, at $t=100 \mathrm{~s}$. The perturbation results, $L^{p}$ and $\hat{w}^{p}$, are indicated by the dashed lines

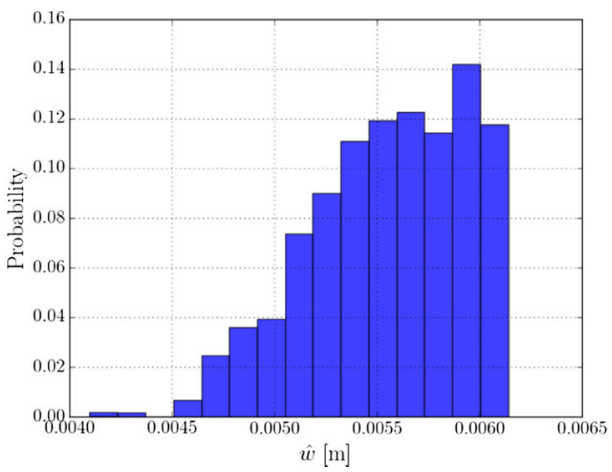

(b) Maximum aperture, $\hat{w}$

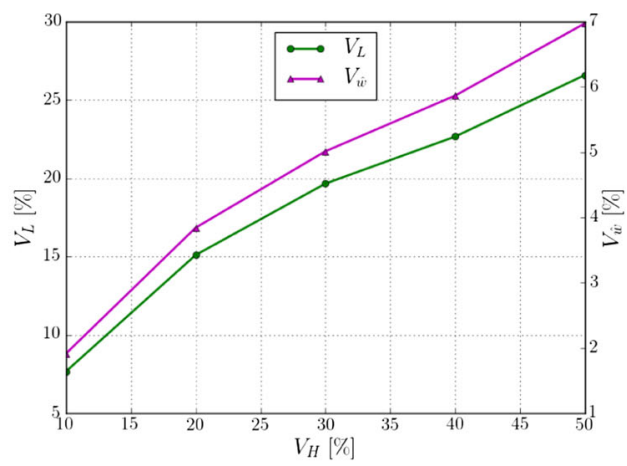

(b) $t=500 \mathrm{~s}$

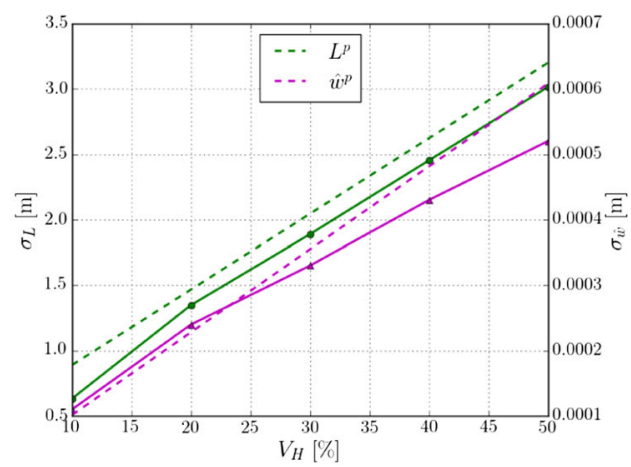

(b) Standard deviation

Table 3 Statistical moments of the fracture length, $L$, and the maximum aperture, $\hat{w}$, at $t=100 \mathrm{~s}$ corresponding to a variation of the plane strain modulus of $V_{E^{\prime}}=50 \%$ for random fields with varying correlation lengths, $l_{E^{\prime}}$

\begin{tabular}{lllllll}
\hline$l_{E^{\prime}}[\mathrm{m}]$ & $\mu_{L}[\mathrm{~m}]$ & $\sigma_{L}[\mathrm{~m}]$ & $V_{L}[\%]$ & $\mu_{\hat{w}}[\mathrm{~m}]$ & $\sigma_{\hat{w}}[\mathrm{~m}]$ & 0.0030 \\
\hline 5 & 27.545 & 6.682 & 24.3 & 0.0071 & 0.0041 & $V_{\hat{w}}[\%]$ \\
10 & 28.496 & 7.177 & 25.2 & 0.0078 & 0.0075 & 0.0037 \\
15 & 29.319 & 6.204 & 21.2 & 0.0074 & 0.0029 \\
20 & 29.735 & 6.601 & 22.2 & 0.0067 & 0.0021 & 39.3 \\
25 & 31.091 & 6.158 & 19.8 & 0.0056 & 0.0005 \\
$\infty$ & 33.619 & 3.348 & 9.9 & & 31.3 \\
\hline
\end{tabular}

Note that the case of $l_{E^{\prime}}=\infty$ corresponds to the homogeneous case discussed in Section 5.3.1 


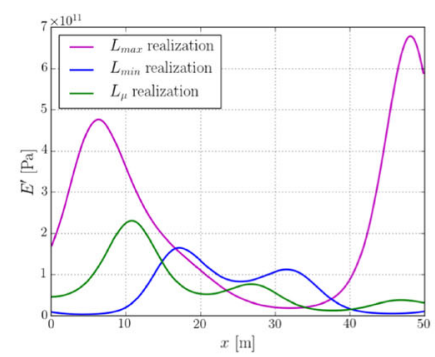

(a1)

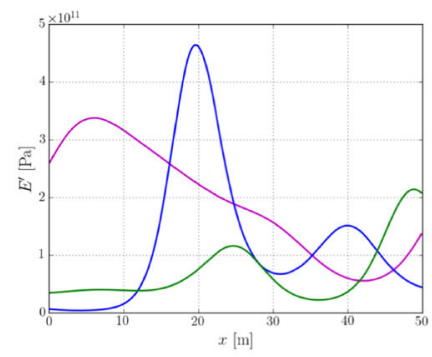

(a2)

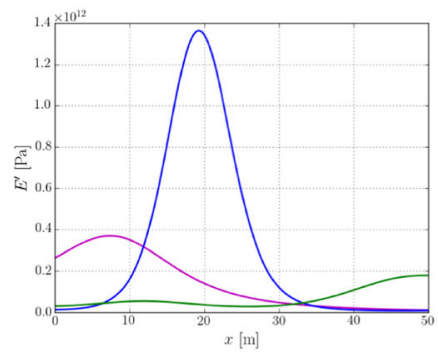

(a3)

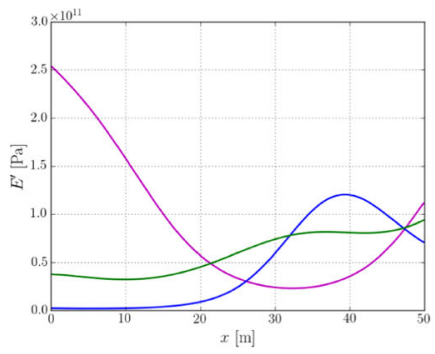

(a4)

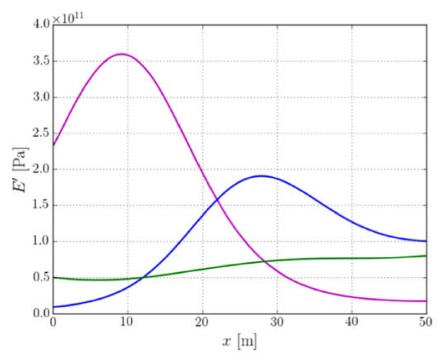

(a5)

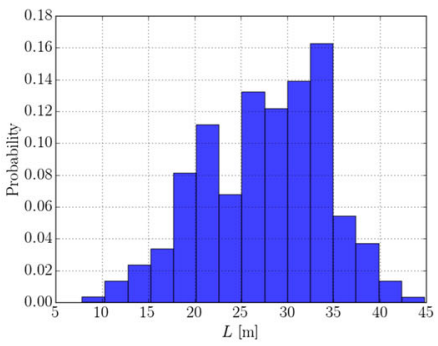

(b1)

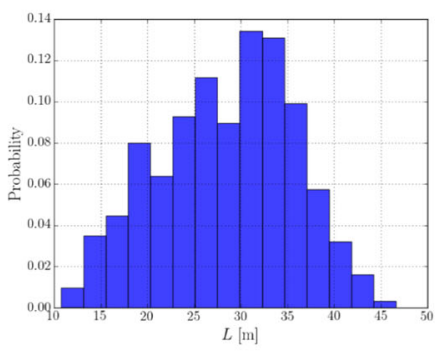

(b2)

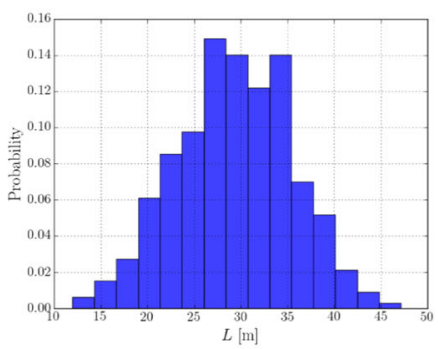

(b3)

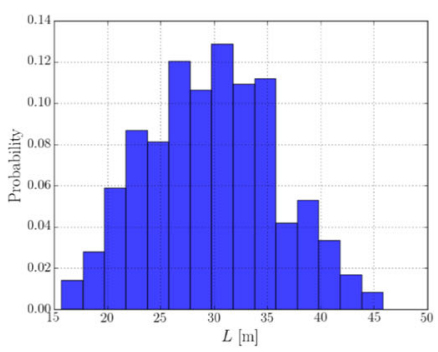

(b4)

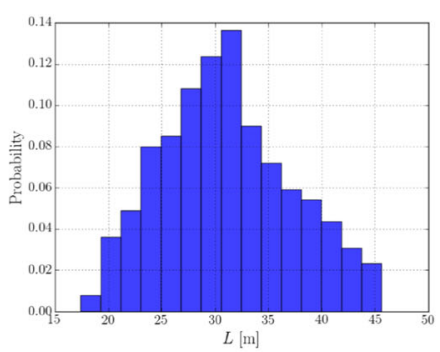

(b5)

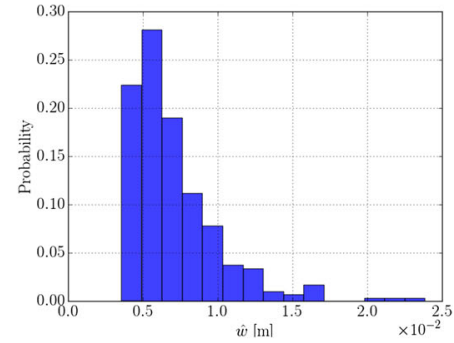

$(\mathrm{c} 1)$

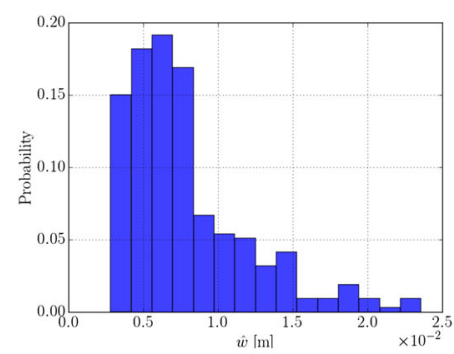

(c2)

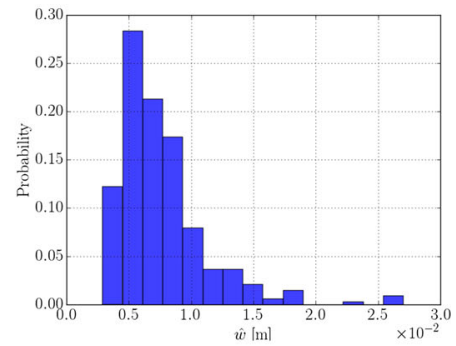

(c3)

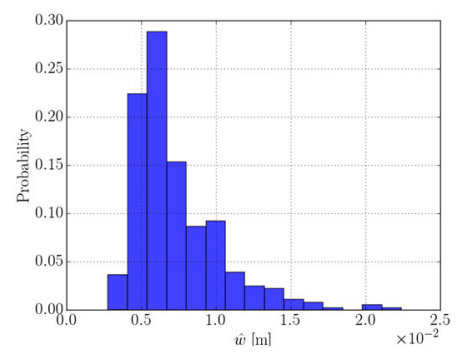

(c4)

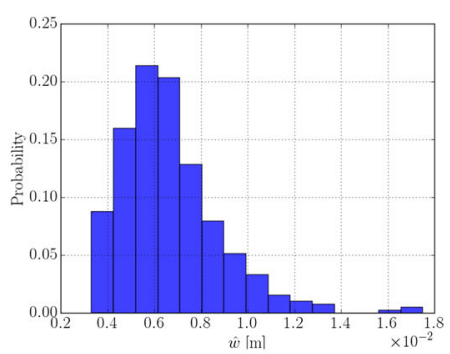

(c5)
Fig. 25 a1-a5 Examples of realization for $l_{E^{\prime}}=(5,10,15,20,25) \mathrm{m}$, respectively. b1-b5 Histograms of the fracture length for $l_{E^{\prime}}=$
$(5,10,15,20,25) \mathrm{m}$, respectively. c1-c5 Histograms of the maximum aperture for $l_{E^{\prime}}=(5,10,15,20,25) \mathrm{m}$, respectively 
pertains to the local elasticity relation in the PKN model, which restricts its application to low-frequency spatial variations of the model parameters.

In order to compute high fidelity solutions that do not pollute stochastic analyses with numerical errors, a moving-mesh finite element method is developed. The employed backward-Euler time integration scheme is supplemented with a sub-iteration technique, such that the mesh propagation relation becomes implicit. The nonlinearity of the model is solved using Newton iterations. We have performed detailed mesh size and time integration step convergence studies. We have found that in order to attain solutions with acceptable accuracy in the context of the stochastic analysis, the finite element methodology had to be enhanced in two manners. First, the global conservation of volume was found to be significantly violated due to the highly non-linear character of the model. The observed loss of volume led to significant underestimation of the fracture length. To circumvent this problem, volume conservation was enforced explicitly by means of a Lagrange multiplier approach. Second, the weakly singular behavior of the fracture opening and pressure at the tip was found to be troublesome in the case of a standard finite element basis. On one hand, the improper representation of the singularity by the basis required the use of an ad hoc tip velocity relation. On the other hand, the mesh resolution of the uniform finite element mesh was found to be insufficient. These issues were resolved by enrichment of the standard finite element space with a singular tip function. We established that in the deterministic setting, our finite element simulations show very good agreement with results reported in literature for a realistic test case even on relatively coarse meshes.

The sensitivity of the fracture evolution process with respect to various random input parameters was studied. From the direct Monte-Carlo simulations, it was found that the mean and standard deviation of the fracture length and fracture mouth opening correspond well to those values obtained using perturbation theory. This observation conveys that -at least for the test case consideredlinearization of the model provides meaningful information on the behavior of the stochastic moments, despite the complexity of the model and its solution procedure.

To demonstrate the suitability of the developed methodology for studying random heterogeneities in formation properties, we have considered a test case in which the formation stiffness was described by a random field. The random dimension was discretized using a KarhunenLoéve expansion. The sampling results demonstrate that the response uncertainty is amplified by the heterogeneous character of the random material property field. For the fracture length, this is explained by the fact that fracture propagation is sensitive to local variations in the elastic properties of the formation because locally compliant regions can inhibit transfer of the fracturing fluid and, hence, propagation of the crack. For the fracture mouth opening an even stronger amplification is observed as a consequence of the fact that the fracture opening is directly related to the material property. Although this observation can be explained well based on the structure of the PKN model, it requires further study to understand to what extend a similar conclusion can be drawn for more sophisticated hydraulic fracturing models.

Although the results presented herein provide fundamental insight into the primary characteristics of the stochastic behavior of the hydraulic fracturing process, it is evident that more detailed information can be obtained by more versatile models and simulation strategies. In particular, the PKN model does not rely on a fracture mechanics model based on the material's fracture toughness, which restricts the scope of this work to fractures in the viscositydominated regime. When considering uncertainty quantification using physically richer models, it will remain key to not pollute the results with numerical errors, which will inevitably lead to computationally complex MonteCarlo methods. The use of alternative stochastic techniques, such as the perturbation method can be expected to yield meaningful results at a much lower cost than direct Monte-Carlo sampling.

Acknowledgements We acknowledge the support from the European Commission EACEA Agency, Framework Partnership Agreement Erasmus Mundus Action 1b, as a part of the EM Joint Doctorate Simulation in Engineering and Entrepreneurship Development (SEED). The work of S. Zlotnik and P. Díez was funded by the project DPI201785139-C2-2-R of the Spanish Ministry and by grant 2017-SGR-1278 from the Generalitat de Catalunya.

Funding information The work of S. Zlotnik and P. Díez was funded by the project DPI2017-85139-C2-2-R of the Spanish Ministry and by grant 2017-SGR-1278 from the Generalitat de Catalunya.

Open Access This article is distributed under the terms of the Creative Commons Attribution 4.0 International License (http:// creativecommons.org/licenses/by/4.0/), which permits unrestricted use, distribution, and reproduction in any medium, provided you give appropriate credit to the original author(s) and the source, provide a link to the Creative Commons license, and indicate if changes were made.

\section{Appendix: Benchmark results}

In Section 5.1, we have considered the deterministic benchmark result based on the case study by Warpinski et al. [41]. The parameters for the considered simulation can be found in Table 1. Figure 5 in Section 5.1 shows the results for this benchmark case as obtained using the finite element technique developed in this manuscript, as well as the results 
Table 4 Numerical output of the model considered in this manuscript formatted consistently with the results in Ref. [41]

\begin{tabular}{llll}
\hline $\begin{array}{l}\text { Time } \\
t[\mathrm{~s}]\end{array}$ & $\begin{array}{l}\text { Fracture height } \\
H[\mathrm{~m}]\end{array}$ & $\begin{array}{l}\text { Fracture length } \\
L[\mathrm{~m}]\end{array}$ & $\begin{array}{l}\text { Maximum aperture } \\
\hat{w}[\mathrm{~m}]\end{array}$ \\
\hline 0 & 51.8 & 2.0000 & 0.0037 \\
1200 & 51.8 & 246.1651 & 0.0089 \\
2400 & 51.8 & 421.3913 & 0.0101 \\
3600 & 51.8 & 575.4035 & 0.0109 \\
4800 & 51.8 & 716.5376 & 0.0114 \\
6000 & 51.8 & 848.7358 & 0.0119 \\
7200 & 51.8 & 974.0853 & 0.0122 \\
8400 & 51.8 & 1093.9648 & 0.0126 \\
9600 & 51.8 & 1209.3184 & 0.0129 \\
10400 & 51.8 & 1320.8104 & 0.0131 \\
12000 & 51.8 & 1428.9438 & 0.0134 \\
\hline
\end{tabular}

for the simulators included in Ref. [41]. Note that the results of these simulators have been reported with intervals of 1200 s. For completeness, in Table 4, we report the results obtained by the method proposed herein with a mesh size of $\Delta x=1 \mathrm{~m}$ and a time step size of $\Delta t=1 \mathrm{~s}$. Note that the presented results have been rounded off to 4 decimals.

Publisher's Note Springer Nature remains neutral with regard to jurisdictional claims in published maps and institutional affiliations.

\section{References}

1. Adachi, J., Detournay, E.: Self-similar solution of a plane-strain fracture driven by a power-law fluid. Int. J. Numer. Anal. Methods Geomech. 26(6), 579-604 (2002)

2. Adachi, J., Siebrits, E., Peirce, A., Desroches, J.: Computer simulation of hydraulic fractures. Int. J. Rock Mech. Mining Sci. 44(5), 739-757 (2007)

3. Adachi, J.I., Peirce, A.P.: Asymptotic analysis of an elasticity equation for a finger-like hydraulic fracture. J. Elast. 90(1), 43-69 (2008)

4. Advani, S., Lee, J.: Finite element model simulations associated with hydraulic fracturing. Society of Petroleum Engineers Journal (1982)

5. Barker, E., Barker, W., Burr, W., Polk, W., Smid, M.: Recommendation for key management part 1: General (revision 3). NIST Spec. Publ. 800(57), 1-147 (2012)

6. Batchelor, G.K.: An Introduction to Fluid Dynamics. Cambridge University Press (2000)

7. Detournay, E.: Mechanics of hydraulic fractures. Annual Review of Fluid Mechanics (2016)

8. England, A.H., Green, A.E.: Some two-dimensional punch and crack problems in classical elasticity. Math. Proc. Camb. Philos. Soc. 59(2), 489-500 (1963)

9. Ganis, B., Mear, M.E., Sakhaee-Pour, A., Wheeler, M.F., Wick, T.: Modeling fluid injection in fractures with a reservoir simulator coupled to a boundary element method. Comput. Geosci. 18(5), 613-624 (2014)
10. Garagash, D., Detournay, E.: The tip region of a fluid-driven fracture in an elastic medium. Trans.-Amer. Soc. Mech. Eng. J. Appl. Mech. 67(1), 183-192 (2000)

11. Geertsma, J., De Klerk, F., et al.: A rapid method of predicting width and extent of hydraulically induced fractures. J. Petrol. Tech. 21(12), 1-571 (1969)

12. Gordeliy, E., Peirce, A.: Coupling schemes for modeling hydraulic fracture propagation using the xfem. Comput. Methods Appl. Mech. Eng. 253, 305-322 (2013)

13. Gutiérrez, M., Krenk, S.: Stochastic Finite Element Methods, chap. 20, pp. 1-25. Wiley, New York (2017)

14. Hanson, M.E., Shaffer, R.J., Anderson, G.D., et al.: Effects of various parameters on hydraulic fracturing geometry. Soc. Pet. Eng. J. 21(04), 435-443 (1981)

15. Howard, G.C., Fast, C.R.: API-57-261, chap. Optimum Fluid Characteristics for Fracture Extension. American Petroleum Institute, New York (1957)

16. Kemp, L.: Study of Nordgren's Equation of Hydraulic Fracturing. SPE Production Engineering (1990)

17. Kenney, J.F.: Mathematics of Statistics. D Van Nostrand Company Inc, Toronto (2013). Affiliated East-West Press Pvt-Ltd; New Delhi

18. Kiureghian, A.D., Liu, P.: Structural reliability under incomplete probability information. J. Eng. Mech. 112(1), 85-104 (1986)

19. Kovalyshen, Y., Detournay, E.: A reexamination of the classical PKN model of hydraulic fracture. Transp. Porous Media 81, 317-339 (2010)

20. Lecampion, B.: An extended finite element method for hydraulic fracture problems. Commun. Numer. Methods Eng. 25(2), 121133 (2009)

21. Lowengrub, M.: A note on Griffith cracks. Proc. Edinb. Math. Soc. 15(2), 131-134 (1966)

22. Meakin, P., Li, G., Sander, L., Louis, E., Guinea, F.: A simple twodimensional model for crack propagation. J. Phys. A Math. Gen. 22(9), 1393 (1989)

23. Melenk, J.M., Babuška, I.: The partition of unity finite element method: basic theory and applications. Comput. Methods Appl. Mech. Eng. 139(1-4), 289-314 (1996)

24. Miehe, C., Mauthe, S.: Phase field modeling of fracture in multiphysics problems. Part III. Crack driving forces in hydro-poroelasticity and hydraulic fracturing of fluid-saturated porous media. Comput. Methods Appl. Mech. Eng. 304, 619-655 (2016)

25. Mikelic, A., Wheeler, M., Wick, T.: Phase-field modeling of a fluid-driven fracture in a poroelastic medium. Comput. Geosci. 19(6), 1171-1195 (2015)

26. Munjiza, A., Owen, D., Bicanic, N.: A combined finite-discrete element method in transient dynamics of fracturing solids. Eng. Comput. 12(2), 145-174 (1995)

27. Neyman, J.: Outline of a theory of statistical estimation based on the classical theory of probability. Philos. Trans. R. Soc. London. Series A Math. Phys. Sci. 236(767), 333-380 (1937)

28. Ng, A.K., Small, J.C.: A case study of hydraulic fracturing using finite element methods. Can. Geotech. J. 36(5), 861-875 (1999)

29. Nordgren, R.: Propagation of vertical hydraulic fractures. Society of Petroleum Engineers Journal (1972)

30. Peirce, A., Siebrits, E.: An Eulerian Finite Volume Method for Hydraulic Fracture Problems. Finite Volumes for Complex Applications IV, ISTE. London, pp. 655-664 (2005)

31. Perkins, T.K., Kern, L.R.: Widths of hydraulic fractures. Journal of Petroleum Technology (1961)

32. Remij, E.W., Remmers, J.J.C., Huyghe, J.M., Smeulders, D.M.J.: The enhanced local pressure model for the accurate analysis of fluid pressure driven fracture in porous materials. Comput. Methods Appl. Mech. Eng. 286, 293-312 (2015) 
33. Remij, E.W., Remmers, J.J.C., Huyghe, J.M., Smeulders, D.M.J.: On the numerical simulation of crack interaction in hydraulic fracturing. Comput. Geosci. 22(1), 423-437 (2018)

34. Settari, A., Cleary, M.P., et al.: Development and testing of a pseudo-three-dimensional model of hydraulic fracture geometry. SPE Prod. Eng. 1(06), 449-466 (1986)

35. Simonson, E., Abou-Sayed, A., Clifton, R., et al.: Containment of massive hydraulic fractures. Soc. Pet. Eng. J. 18(01), 27-32 (1978)

36. Sneddon, I.N.: The distribution of stress in the neighbourhood of a crack in an elastic solid. Proc. R. Soc. London A: Math. Phys. Eng. Sci. 187(1009), 229-260 (1946)

37. Sneddon, I.N.: A note on the problem of the penny-shaped crack. Math. Proc. Camb. Philos. Soc. 61(2), 609-611 (1965)

38. Spanos, P.D., Ghanem, R.: Stochastic finite element expansion for random media. J. Eng. Mech. 115(5), 1035-1053 (1989)

39. Valko, P., Economides, M.: Hydraulic Fracture Mechanics. Wiley (1995)

40. Wangen, M.: Finite element modeling of hydraulic fracturing on a reservoir scale in 2d. J. Pet. Sci. Eng. 77(3), 274-285 (2011)

41. Warpinski, N., Abou-Sayed, I., Moschovidis, Z., Parker, C.: Hydraulic fracture model comparison study: complete results. Tech. rep., Sandia National Labs., Albuquerque, NM (United States); Gas Research Inst., Chicago, IL (United States (1993)
42. Wheeler, M.F., Wick, T., Wollner, W.: An augmented-Lagrangian method for the phase-field approach for pressurized fractures. Comput. Methods Appl. Mech. Eng. 271, 69-85 (2014)

43. Wilson, Z.A., Landis, C.M.: Phase-field modeling of hydraulic fracture. J. Mech. Phys. Solids 96, 264-290 (2016)

44. Youn, D., Griffiths, D.: Stochastic analysis of hydraulic fracture propagation using the extended finite element method and random field theory. In: Integrating Innovations of Rock Mechanics: Proceedings of the 8th South American Congress on Rock Mechanics, 15-18 November 2015, p. 189. IOS Press, Buenos Aires (2015)

45. Zhang, G., Liu, H., Zhang, J., Wu, H., Wang, X.: Threedimensional finite element simulation and parametric study for horizontal well hydraulic fracture. J. Pet. Sci. Eng. 72(3), 310-317 (2010)

46. Zhao, H., Li, Z., Zhu, C., Ru, Z.: Reliability analysis models for hydraulic fracturing. J. Pet. Sci. Eng. 162, 150-157 (2018)

47. Zheltov, A.K. et al.: 3 . Formation of vertical fractures by means of highly viscous liquid. In: 4th World Petroleum Congress. World Petroleum Congress (1955)

48. Zimmerman, R., In-Wook, Y.: Fluid Flow in Rock Fractures: From the Navier-Stokes Equations to the Cubic Law, pp. 213-224. American Geophysical Union (AGU) (2013) 